\title{
Environmental factors driving arbuscular mycorrhizal fungal communities associated with endemic woody plant Picconia azorica on native forest of Azores
}

\author{
Catarina Drumonde Melo ${ }^{1,2,3}$ (D) $\cdot$ Christopher Walker ${ }^{4,5} \cdot$ Claudia Krüger $^{6} \cdot$ Paulo A.V. Borges $^{2} \cdot$ Sara Luna $^{1}$. \\ Duarte Mendonça $^{1} \cdot$ Henrique M. A. C. Fonseca ${ }^{7} \cdot$ Artur Câmara Machado $^{1}$
}

Received: 20 August 2019 / Accepted: 8 November 2019 / Published online: 4 December 2019

(C) The Author(s) 2019

\section{Abstract \\ Purpose}

Arbuscular mycorrhizal fungi (AMF) play important key roles in the soil ecosystems as they link plants to the root-inaccessible part of soil. The aims of this study were to investigate which environmental factors influence the spatial and temporal structuring of AMF communities associated to Picconia azorica in two Azorean islands (Terceira and São Miguel islands), and investigate the seasonal variation in AMF communities between the two islands.

\section{Methods}

Communities of AMF associated with P. azorica in native forest of two Azorean islands (Terceira and São Miguel) were characterised by spore morphology or molecular analysis.

\section{Results}

Forty-five AMF spore morphotypes were detected from the four fragments of $P$. azorica forest representing nine families of AMF. Acaulosporaceae (14) and Glomeraceae (9) were the most abundant families. AMF density and root colonisation varied significantly between islands and sampling sites. Root colonisation and spore density exhibited temporal patterns, which peaked in spring and were higher in Terceira than in São Miguel. The relative contribution of environmental factors showed that factors such as elevation, relative air humidity, soil $\mathrm{pH}$, and soil available $\mathrm{P}, \mathrm{K}$, and $\mathrm{Mg}$ influenced AMF spore production and root colonisation.

\section{Conclusion}

Different sporulation patterns exhibited by the members of the commonest families suggested different life strategies. Adaptation to a particular climatic and soil condition and host phenology may explain seasonal differences in sporulation patterns. Cohorts of AMF associated to $P$. azorica are shaped by regional processes including environmental filters such as soil properties and natural disturbance.

Keywords Arbuscular mycorrhizal fungi $\cdot$ Endemic plant $\cdot$ Native forest $\cdot$ Environmental factors $\cdot$ Temporal patterns $\cdot$ Root colonisation $\cdot$ Spore density

Catarina Drumonde Melo

cdrumonde73@gmail.com; cdrumonde@uac.pt

1 CBA-UAç - Biotechnology Center of Azores, Faculty of Agricultural Sciences and Environment, University of Azores, Rua Capitão João D’Avila, São Pedro, 9700-042, Angra do Heroísmo, Terceira, Azores, Portugal

2 Departamento de Ciências e Engenharia do Ambiente, cE3c-Centre for Ecology, Evolution and Environmental Changes/Azorean Biodiversity Group and Universidade dos Açores, Rua Capitão João D'Ávila, São Pedro, 9700-042 Angra do Heroísmo, Terceira, Azores, Portugal
3 CFE - Centre for Functional Ecology, Department of Life Sciences, University of Coimbra, 3001-401 Coimbra, Portugal

4 Royal Botanic Garden Edinburgh, 20A Inverleith Row, Edinburgh EH3 5LR, UK

5 School of Plant Biology, University of Western Australia, 35 Stirling Highway, Crawley, Perth, WA 6009, Australia

6 Institute of Botany, The Czech Academy of Sciences, Zámek 1, 25243 Průhonice, Czech Republic

Department of Biology \& GeoBioTec, University of Aveiro Campus Universitário de Santiago, 3810-193 Aveiro, Portugal 


\section{Introduction}

Arbuscular mycorrhizal fungi (AMF) are mutually beneficial associations between plant roots and soil fungi of the phylum Glomeromycota (Schüßler et al. 2001). This symbiotic relationship facilitates plant growth through enhancing uptake of several macro- and micro-nutrients of low mobility (e.g., P, $\mathrm{Zn}, \mathrm{Cu}$ ) in soil (Porras-Soriano et al. 2009; Bati et al. 2015). Arbuscular mycorrhizas can also provide other ecological advantages such as influencing microbial and chemical environment of the mycorrhizosphere (Wang et al. 2007; Trabelsi and Mhamdi 2013), stabilising soil aggregates (Rillig and Mummey 2006; Willis et al. 2013) and conferring plant tolerance to several abiotic and biotic stresses (Hildebrandt et al. 2007; Pozo and Azcón-Aguilar 2007). Several studies have pointed out that communities of AMF occurring in different ecosystems have different species composition (Öpik et al. 2006; Zhao et al. 2017), and their distributions may be products of environment, interspecific competition and regional spatial dynamics (Lekberg et al. 2007). Recent studies also suggest that land management can impact the diversity and distribution of AMF. More intensively, conventionally managed farm soils exhibit lower diversity and greater similarity of AMF communities than less intensively organically managed soil (van der Gast et al. 2011; Melo et al. 2014).

Seasonal fluctuations such as rainfall patterns (Hazard et al. 2013; Alguacil et al. 2015) and temperature (Dumbrell et al. 2011) influence AMF development in the soil. Levels of available N (Egerton-Warburton et al. 2007; Velázquez et al. 2018), P (Bainard et al. 2014; Zhao et al. 2017), soil pH (Melo et al. 2017), soil moisture (Deepika and Kothamasi 2015) and organic matter (OM) (Alguacil et al. 2014; Wang et al. 2015) can influence AMF communities.

The Azores archipelago has some ecosystems with high diversity of endemic plants species. The native forest of the Azores, which is one such reservoir of biodiversity, includes the so-called Laurisilva (Elias et al. 2016) covering less than $10 \%$ of the total area of the archipelago, and being almost absent in some islands (e.g., Graciosa, Corvo) due to human activities (Borges et al. 2005).

Picconia azorica (Tutin) Knobl. (Oleaceae), locally named "pau-branco", is an endangered species endemic to Laurisilva forest, present in all the islands except Graciosa (Silva et al. 2009, 2010), being present in coastal and medium elevation forests, coastal cliffs, ravines, lava flows, coastal scrubland (with Erica azorica, Morella fava; Picconia-Morella" Lowland forest sensu Elias et al. 2016) and natural forests (with Morella fava, Laurus azorica; "Laurus submontane forests" sensu Elias et al. 2016). Because of habitat degradation, it is a priority Azorean endemic species for conservation, listed as endangered (EN B1 + 2c) on the IUCN Red List 2004, and in Annex II of the EC Habitats Directive. Habitat degradation by expansion of agricultural land, forestation, competition by alien species and isolation of populations (Silva et al. 2009) threaten P. azorica populations. Vegetative and seed propagation (Martins et al. 2011, 2012), and knowledge of population genetics (Martins et al. 2013) have been improved. However, to design effective conservation strategies or recovery plans, improved knowledge of plant distribution, population number, population size and the ecological constraints to species establishment is needed (Brigham and Schwartz 2003).

The use of native or indigenous mycorrhizas from soil can benefit restoration programs, by improving plant nutrition and performance under environmental stress and facilitating plant adaptation in both nursery and field conditions (Graham et al. 2013; Maltz and Treseder 2015). Selection of appropriate AMF and production of local inocula, in sufficient quality and quantity are critical issues for their application in agriculture, because native AMF isolates appear to be physiologically and genetically adapted to the stress conditions of the target environment and to their native host (de Oliveira et al. 2017). Two previous studies detail native AMF communities and the mycorrhizal status of Juniperus brevifolia and Picconia azorica in Azorean native forest (Melo et al. 2017, 2018) which phylogenetic analysis showed that at least 18 species were present in the root zone of $P$. azorica, but different patterns of richness and distribution between islands (Terceira and São Miguel) suggested that geographical and historical factors are determining shaping of AMF communities in native forest of Azores. Here, we discuss likely causes of these differences. However, which factors contribute to shape AMF composition with reference to specific host species under different environmental conditions are still unknown in Azorean native forest. AMF are ecologically and functionally distinct and vary in their response to changes in the abiotic environment (Van der Heijden and Scheublin 2007; Soka and Ritchie 2014). Consequently, the knowledge of the mycorrhizal condition on soil and their variability over time is essential to determine different sporulation patterns, and the variation in the occurrence of species in different AMF families. This would enable the selection of functionally complementary species, or their combinations, which could be crucial for using AM technology in the survival of endemic plants under field conditions.

The objective of this study are to investigate which environmental factors influence the spatial and temporal structuring of AMF communities associated to P. azorica in two Azorean islands (Terceira and São Miguel islands) and determinate if there was the seasonal differences in AMF communities between the two islands. 


\section{Material and methods}

\section{Study sites}

The Azores have a temperate oceanic climate characterised by high levels of relative atmospheric humidity (up to $95 \%$ in high elevation native forests), ensuring moderate thermal variations throughout the year (Azevedo et al. 1999). The average temperature is $17.5^{\circ} \mathrm{C}$ in low elevations, with the maximum temperature $\left(26{ }^{\circ} \mathrm{C}\right)$ in August and minimum $\left(10^{\circ} \mathrm{C}\right)$ in February (Azevedo et al. 1999). Precipitation reaches its peak in January-February and is at a minimum in July (Azevedo 1996): Angra do Heroísmo (47 m), $969 \mathrm{~mm}$ year (140 mm in January and $40 \mathrm{~mm}$ in July); and Serra de S. Bárbara (1023 m), $3000 \mathrm{~mm}$ year (Azevedo 1996).

This study was conducted in two Islands of the Azorean archipelago, São Miguel (4.01 Myr old) and Terceira (4 Myr old) (Ávila et al. 2016). In Terceira, the native forest include two native fragments of Picconia azorica - Terra Brava (TB) and Serreta (TS) (Melo et al. 2018). Terra Brava is included in the very wet Laurisilva at $650 \mathrm{~m}$ elevation, dominated by endemic woody plants, predominantly Laurus azorica, Ilex perado azorica, Frangula azorica, Vaccinium cylindraceum, Erica azorica, Myrsine africana, and occasionally by Juniperus brevifolia and P. azorica. Serreta is at low elevation $(160 \mathrm{~m})$ and is characterised by a low diversity of plants, mainly by Morella faya and P. azorica, and occasionally by L. azorica. These forests are located in the most thermophilic areas of Azores and are almost extinct (Dias 1996). The highest canopy is dominated by a dense cover of Pittosporum undulatum, and rarely by L. azorica. This forest is mixed with other invasive woody. species including Metrosideros excelsa, Eucalyptus globulus, Acacia melanoxylon, Sphaeropteris cooperi, Fuchsia magellanica and Rubus inermis. The herbaceous stratum is dominated by Dryopteris azorica, Hedera helix var. azorica, Smilax aspera and Gomphocarpus fruticosus (Dias 1996). In São Miguel, the two native fragments of P. azorica - Lombo Gordo (SG) and Ribeira Quente (SR) are located at low lands (Melo et al. 2018). Lombo Gordo (95 m elevation) is covered by a coastal scrubland where $P$. azorica dominates in certain areas but is mixed with other native and invasive woody species including M. faya, E. azorica, P. undulatum, Arundo donax, Hedychium gardnerianum and Phormium tenax (Martins et al. 2011). Ribeira Quente (87 m elevation) is also a coastal scrubland dominated by the endemic plants L. azorica and P. azorica, but also with other native and invasive woody species such as P. undulatum, M. Faya and A. melanoxylon.

\section{Sample collection and pot culture establishment}

On the three sampling periods, September 2012 (autumn 2012), May 2013 (spring 2013) and September 2013 (autumn), 10 soil samples with associated plant roots were collected on each native fragments of $P$. azorica from Terceira (Terra Brava - TB; and Serreta - TS), and on each native fragments of $P$. azorica from São Miguel (Lombo Gordo - SG; and Ribeira Quente - SR), resulting in 120 soil samples $(2$ islands $\times 2$ sites/island $\times 10$ samples/site $\times 3$ sampling dates). The distance between samples taken on each site was a minimum $25 \mathrm{~m}$ and maximum $40 \mathrm{~m}$, and the distance between sample sites was about $20 \mathrm{~km}$ in Terceira, and $15 \mathrm{~km}$ in São Miguel.

Each soil sample was geo-referenced and consisted of four subsamples collected from four different points (approximately N, S, E, and W) around each $P$. azorica plant, which were pooled. Approximately $2 \mathrm{~kg}$ of soil was collected from the rooting zone of each $P$. azorica to a depth of $20-30 \mathrm{~cm}$ with a shovel; vegetation cover was removed during sampling and replaced afterwards. The soil samples were air-dried, sieved through a $2-\mathrm{mm}$ mesh, and stored at $4{ }^{\circ} \mathrm{C}$ before analysis. Subsequent samples were taken from the same marked plants following the cardinal points.

\section{Spore identification}

Two open pot trap cultures (Gilmore 1968) were established from each sample, one with 1-week-old Zea mays seedlings, and the other with micropropagated $P$. azorica seedlings (Melo et al. 2014, 2017, 2018). Seeds of $Z$. may were surface sterilised by immersion in alcohol $(96 \%)$ for $30 \mathrm{~s}, 4 \%$ household bleach for $2 \mathrm{~min}$, followed by two rinses in sterile distilled water. Seeds were sown in trays on autoclaved volcanic soil and germinated in the greenhouse. Micropropagated $P$. azorica seedlings were derived from a single clone of the in vitro collection of CBA-UAc, previously acclimatised for 2 months in the greenhouse. One seedling of each host plant was planted into each pot, containing $1.5 \mathrm{~kg}$ of fresh soil sampled from each site mixed with autoclaved volcanic soil $(2: 1, v / v)$. Ten pots per sampling site were established, resulting in 240 open-pot cultures $(4$ sites $\times 10$ samples/site $\times 3$ sampling dates $\times 2$ trap cultures). All pots were maintained in a greenhouse for 5 months and watered every 2 days with distilled water.

Glomeromycotan spores were extracted from $50 \mathrm{~g}$ of airdried soil of each sample from field soil and trap cultures by wet sieving and sucrose centrifugation (Walker 1992) and were stored at $4{ }^{\circ} \mathrm{C}$ in autoclaved water pending examination. Different spore types were initially separated, in water, under a stereo-microscope. Representatives of each morphotype were identified through a compound microscope in a 4:1 mixture of polyvinyl alcohol lacto-glycerol (PVLG) and Melzer's reagent, photographed and stored as semi-permanent slide preparations. The morphotypes were counted under a dissecting microscope after classification into either known species, or types that could not be placed in a current species, based on colour, size, surface ornamentation, hyphal attachment, 
reaction to Melzer's reagent and wall structure. Identification of spores was carried out by use of primary literature and experience from more than 40 years of taxonomic study of the Glomeromycota by C. Walker (e.g., Walker and Trappe 1981; Walker et al. 1984; Koske and Walker 1985; Walker et al. 1986; Walker and Diederichs 1989; Walker and Vestberg 1998; Schüßler et al. 2011; Krüger et al. 2012; Redecker et al. 2013; Schüßler and Walker 2019), and joint authorship with A. Schüßler of the website amf-phylogeny. com, which lists all accepted species in the phylum. The illustrated manual of Blaszkowski (2012) was also used.

Single species culture attempts of the different AM fungal morphotypes extracted from the field soil and trap cultures were established in pots using river sand as growing substrate and Plantago lanceolata as host plant. Spores with a healthy appearance (oily contents; without evidence of contamination by non-AMF) of each AM fungal morphotype were used as inoculum by placing them on the plant root system under a dissecting microscope before transplanting into the pot (Melo et al. 2017, 2018). Individuals of morphologically characterised spore types extracted from field soil, trap cultures or single spore cultures were used for DNA analysis. Information about molecular characterisation, including DNA extraction, PCR, cloning, RFLP, sequencing and phylogenetic analyses are published in Melo et al. (2018).

\section{Mycorrhizal colonisation assessment}

The collected roots were stores in $50 \%$ ethanol pending processing. Root fragments of approximately $1 \mathrm{~cm}$ were cleared in $10 \%(w / v) \mathrm{KOH}$ by heating at $90{ }^{\circ} \mathrm{C}$ for 60 to $90 \mathrm{~min}$, depending on the degree of lignification of the roots, to empty cells of their cytoplasmic contents to improve the observation of the symbiotic fungus. After that, root fragments were rinsed with water to remove all traces of $\mathrm{KOH}$, and then immersed in $10 \%(v / v)$ commercial bleach until roots are clarified. Subsequently, they were left to acidify overnight in $1 \% \mathrm{HCl}$. Staining was with blue ink (Parker Quink 1\%) for $30 \mathrm{~min}$ at 60 ${ }^{\circ} \mathrm{C}$, followed by distaining in acidified glycerol. The amount of colonisation was estimated using a grid-intersect method with 100 intersects under a compound microscope at $\times 200$ magnification (McGonigle et al. 1990). Root-intersects that contained vesicles, arbuscules or hyphae were scored as mycorrhizal. The decision to score hyphae as mycorrhizal was based on the associated presence of vesicles, arbuscules, spores and the morphology of the mycelium. In total, 120 samples were examined to score 100 intersections in each sample.

\section{Estimation of climate data}

Climate data based on the mean values of temperature and rainfall for each sampling month (Sep 2012, May 2013 and
Sep 2013) were estimated for $P$. azorica native fragments by the Insular climate at local scale model (CIELO) model (Azevedo 1996), which has been calibrated and validated for the Terceira and São Miguel Azores islands. The climatic environmental variables of the CIELO model are related to site regional scale characteristics and thus apply to all samples within each plot.

\section{Soil analysis}

The soil analyses (Table 1) were performed for each individual soil sample collected during the three sampling dates at the University of Azores Soil Laboratory (CITA-A). Potassium $(\mathrm{K})$, calcium $(\mathrm{Ca})$ and magnesium $(\mathrm{Mg})$ were extracted with sodium acetate (1/5) at $\mathrm{pH} 7$ and determined using a Varian ICP atomic emission spectrophotometer. Soil $\mathrm{pH}$ was measured from a soil and water paste $(1: 2.5 \mathrm{v} / \mathrm{v})$, and available phosphorus (P) (Olsen and Sommers 1982) by atomic absorption spectrometry after extraction with a $0.5 \mathrm{M} \mathrm{NaHCO}_{3}$ solution at $\mathrm{pH}$ 8.5. Total soil nitrogen $(\mathrm{N})$ (Kjehldahl) content (Allen 1989) and organic matter (OM) (by dry-ashing) were also measured.

\section{Data analyses}

Spore density, richness and occurrence were based on spores recovered directly from the soil samples. The terms are used in the following meaning:

- Spore richness: number of taxa per sample.

- Spore density: number of spores of each taxon per $50 \mathrm{~g}$ of dried soil.

- Frequency of occurrence (FR): percentage of samples containing a particular taxon or morphotype among all samples, reflecting the distribution status.

- Relative spore density (RD): ratio of spore density of a particular taxon or morphotype to the total density of spores, showing comparing sporulation of different morphotypes in a particular soil.

- The importance value (IV): evaluation of the dominance of AMF species based on FR and RD, calculated as IV = $(\mathrm{FR}+\mathrm{RD}) / 2$. An IV $\geq 50 \%$ indicates that a taxon (genus or species) is dominant; $10 \%<\mathrm{IV}<50 \%$ applies to common taxa; and IV $\leq 10 \%$ indicates that a taxon is rarely recovered (Chen et al. 2012; Abdelhalim et al. 2013).

Analysis among native fragments followed a hierarchical nested design, with the forest fragments nested within each island and sampling time nested within fragments. Variation in spore density between islands, fragments within each island and sampling time within each fragment were analysed by Nested ANOVA followed by a Tukey honestly significant difference test at $p<0.05$ (Minitab 2000). All data were 
Table 1 Edaphic and climatic parameters in the sampling fragments of Terceira (Terra Brava - TB; Serreta - TS) and São Miguel (Lombo Gordo - SG; Ribeira Quente - SR) at three different sampling dates (September 2012, May 2013 and September 2013)

\begin{tabular}{|c|c|c|c|c|}
\hline \multirow[t]{2}{*}{ Parameters ${ }^{\mathrm{a}}$} & \multicolumn{2}{|l|}{ Terceira } & \multicolumn{2}{|l|}{ São Miguel } \\
\hline & TB & $\mathrm{TS}$ & SG & SR \\
\hline $\mathrm{pH}$ & $4.45 \pm 0.04 \mathrm{a}$ & $5.40 \pm 0.10 b$ & $5.87 \pm 0.10 \mathrm{~b}$ & $5.59 \pm 0.08 b$ \\
\hline $\mathrm{N}\left(\mathrm{mg} \mathrm{kg}^{-1}\right)$ & $15.41 \pm 0.97 \mathrm{a}$ & $9.81 \pm 0.97 b$ & $5.09 \pm 0.52 \mathrm{c}$ & $4.76 \pm 0.52 c$ \\
\hline $\mathrm{P}\left(\mathrm{mg} \mathrm{kg}^{-1}\right)$ & $8.59 \pm 0.36 \mathrm{a}$ & $9.10 \pm 0.51 \mathrm{a}$ & $14.59 \pm 1.58 b$ & $13.05 \pm 0.92 b$ \\
\hline $\mathrm{K}\left(\mathrm{mg} \mathrm{kg}^{-1}\right)$ & $130.34 \pm 7.80 \mathrm{a}$ & $170.13 \pm 13.47 \mathrm{a}$ & $320.30 \pm 20.81 b$ & $637.35 \pm 57.16 \mathrm{c}$ \\
\hline $\mathrm{Ca}\left(\mathrm{mg} \mathrm{kg}^{-1}\right)$ & $392.99 \pm 46.82 \mathrm{a}$ & $1108.37 \pm 129.20 b$ & $862.95 \pm 59.59 \mathrm{~b}$ & $1136.72 \pm 141.76 b$ \\
\hline $\operatorname{Mg}\left(\mathrm{mg} \mathrm{kg}^{-1}\right)$ & $265.02 \pm 19.22 \mathrm{a}$ & $570.10 \pm 38.26 b$ & $617.70 \pm 32 \pm 79 b$ & $536.82 \pm 47.02 b$ \\
\hline $\mathrm{OM}(\%)$ & $43.65 \pm 2.87 \mathrm{a}$ & $21.10 \pm 2.31 b$ & $10.90 \pm 0.96 \mathrm{c}$ & $9.71 \pm 1.04 \mathrm{c}$ \\
\hline Altitude ( $\mathrm{m}$ a.s.1.) & 650 & 160 & 95 & 87 \\
\hline Average monthly rainfall (mm) & $199.54 \pm 9.98$ & $47.63 \pm 4.13$ & $92.20 \pm 17.43$ & $92.20 \pm 14.47$ \\
\hline Average monthly temperature $\left({ }^{\circ} \mathrm{C}\right)$ & $15.80 \pm 0.43$ & $15.30 \pm 0.41$ & $15.78 \pm 0.53$ & $15.78 \pm 0.44$ \\
\hline Average monthly minimum temperature $\left({ }^{\circ} \mathrm{C}\right)$ & $13.06 \pm 0.44$ & $17.76 \pm 0.40$ & $17.31 \pm 0.55$ & $17.31 \pm 0.45$ \\
\hline Average monthly maximum temperature $\left({ }^{\circ} \mathrm{C}\right)$ & $18.54 \pm 0.42$ & $22.73 \pm 0.51$ & $22.28 \pm 0.62$ & $22.28 \pm 0.52$ \\
\hline Average air relative humidity $(\%)$ & $97.43 \pm 0.07$ & $76.75 \pm 0.33$ & $80.24 \pm 0.30$ & $80.24 \pm 0.25$ \\
\hline
\end{tabular}

${ }^{\text {a }}$ Values are mean values of 30 samples \pm SE. Means followed by the same letter do not differ significantly (Tukey test, $p<0.05$ )

checked for normality, and transformation was attempted if necessary with the aim of fulfilling the assumptions of Nested ANOVA. AMF spore density of species in the commonest families (Acaulosporaceae, Diversisporaceae, Gigasporaceae and Glomeraceae) and mycorrhizal colonisation by different AMF structures (total colonisation, colonisation by arbuscules, intraradical spores or vesicles, and hyphae) were not normally distributed and could not be normalised by transformation to allow parametric analysis. Consequently, non-parametric methods, Kruskal-Wallis one-way analysis of variance by ranks and multiple comparisons between the samples with Kruskal-Wallis with SPSS Version 22 (Corp 2013) were applied.

To evaluate relationships between environmental variables and spore density of the commonest AMF families, and between the mycorrhizal colonisation by different AMF structures were evaluated by linear multiple regression. Differences in soil parameters among sites were tested by one-way analysis of variance, and a Tukey honestly significant difference test at $p<0.05)$. Principal component analysis (PCA) with the $\log (x+1)$ including the soil parameters of each sample in each fragment was used to summarise variation and explore similarities between soils with Community Analysis Package v. 4.0 (pisces-conservation.com) (Seaby et al. 2004). Canonical correspondence analysis (CCA) with CANOCO 4.5 (ter Braak and Smilauer 2002) was used to investigate relationships between soil variables, climate variables and density of each spore morphotype per sample. A reduced soil variable data set based on an evaluation of the Pearson product-moment correlations among spore density and soil variables (Griffith et al. 2001) was used to reduce problems with co-linearity in the multivariate analysis. When pairs of environmental variables were correlated at $r>0.70$, one variable was dropped from the environmental data set. Significance of the CCA analysis was tested using a Monte Carlo permutation test with 1000 unrestricted random permutations of residuals $(p \leq 0.05)$ from a reduced model against the null hypothesis that glomeromycotan spore density was unrelated to the treatments as nominal environmental variables (ter Braak and Wiertz 1994).

\section{Results}

\section{Soil analysis}

The soil from the two islands varied significantly in all the measured chemical properties (Table 1) among the four native fragments, but not at the three sampling times. Soil $\mathrm{pH}$ varied significantly between the four native fragments (one-way ANOVA: $\left.F_{(3)}=63.47, p<0.001\right)$. TB showed the lowest soil $\mathrm{pH}$, and $\mathrm{SG}$ the highest, but there was no significant difference in soil $\mathrm{pH}$ between TS, SR and SG. Soil available N (one-way ANOVA: $F_{(3)}=63.47, p<0.001$ ) and OM content (one-way ANOVA: $\left.F_{(3)}=49.65, p<0.001\right)$ also changed significantly among sites. The highest values of total $\mathrm{N}$ and $\mathrm{OM}$ were observed in TB.

Soil available P (one-way ANOVA: $F_{(3)}=13.50, p<$ 0.001 ) and $\mathrm{K}$ (one-way ANOVA: $F_{(3)}=75.60, p<0.001$ ) varied significantly among native fragments. Concerning the two soil properties the highest values was observed in both São Miguel sites, with no significant differences among them, 
except for the available K, which was higher in SR than in SG. Significant differences were also found in available $\mathrm{Ca}$ (oneway ANOVA: $F_{(3)}=17.36, p<0.001$ ) and $\mathrm{Mg}$ (one-way ANOVA: $\left.F_{(3)}=21.79, p<0.001\right)$ among the four native fragments. For $\mathrm{TB}, \mathrm{Ca}$ and $\mathrm{Mg}$ values were significantly lower than for TS, SG and SR, which were not significantly different from each other.

Principal component analysis showed that TB was most dissimilar from the others, whereas SR and SG were more similar to each other than TB and TS (Fig. 1). Organic matter, nitrogen content and $\mathrm{pH}$ were the main factors contributing to the variance of the samples, and there was a positive relationship between $\mathrm{P}, \mathrm{Ca}$ and $\mathrm{Mg}$ content. The two axes explained $78 \%$ of the variation present in the samples.

\section{Glomeromycotan spore types}

From the 22,736 spores extracted among the 120 rhizosphere soil samples, 45 distinct morphotypes from among nine families were detected (Table 2). The families with most AMF species were Acaulosporaceae (14) and Glomeraceae (9) (Table 2). There were ten different undetermined glomoid morphotypes, but these cannot be placed even to genus, as the glomoid spore form is common to several different higher taxa (for example, members of the Glomeraceae and Diversisporaceae, Ambisporaceae and Paraglomeraceae possess glomoid spores, but belong in different orders). Spores from the Glomeraceae were found in almost all samples (97\%) followed by those from Acaulosporaceae (76\%), Gigasporaceae (53\%) and Diversisporaceae (42\%). Of the 45 morphotypes recorded, 22 occurred only in Terceira, most of which were from the Acaulosporaceae and Glomeraceae. Eighteen spore types were found in both islands, and just five from the Glomeraceae only occurred in São Miguel. In Terceira, especially in TB, Glomoid sp.6 was the dominant species $(\mathrm{RD}=45.34 \%, \mathrm{FR}=73.33 \%, \mathrm{IV}=59.18 \%)$, followed by Ac. brasiliensis $(\mathrm{RD}=11.84 \%, \mathrm{FR}=93.33 \%$, IV $=$ $52.59 \%$ ), while in TS, Glomeraceae sp. 1 was the dominant species $(\mathrm{RD}=45.15 \%, \mathrm{FR}=83.33 \%, \mathrm{IV}=64.24 \%)$ followed by Glomus sp. $2(\mathrm{RD}=35.59 \%, \mathrm{FR}=63.33 \%$, IV $=49.42 \%)$ (Table 3). In São Miguel, Glomeraceae sp. 1 was the dominant species ( $\mathrm{RD}=44.32 \%, \mathrm{FR}=66.67 \%, \mathrm{IV}=55.49 \%)$ in $\mathrm{SG}$, while in $\mathrm{SR}$, the dominant species were Ac. mellea $(\mathrm{RD}=$ $17.5 \%, \mathrm{FR}=9.00 \%, \mathrm{IV}=53.53 \%$ ) followed by Glomus sp. 2 $(\mathrm{RD}=26.77 \%, \mathrm{FR}=8.00 \%, \mathrm{IV}=53.39 \%)($ Table 3$)$.

\section{Spore density}

The AMF species density differed significantly between island (Nested ANOVA: $F_{(1,2)}=51.44, p<0.001$ ), and among sampling dates within native fragment $\left(F_{(8,99)}=9.64, p<\right.$ $0.001)$. However, no significant differences were observed in AMF spore density between native fragments within each island (Nested ANOVA: $F_{(2,4)}=0.11, p=0.114$ ). The average AMF spore density per sample was higher in Terceira than in São Miguel (Fig. 2a) and showed a marked temporal pattern among sampling times, especially between the first autumn and the following spring, with an increased spore density during spring (Fig. 2b). The minimum spore density was found in SG in the first and second Autumn with an average of 31 and 82 spores per $50 \mathrm{~cm}^{3}$, respectively (Fig. 2b), while TS showed

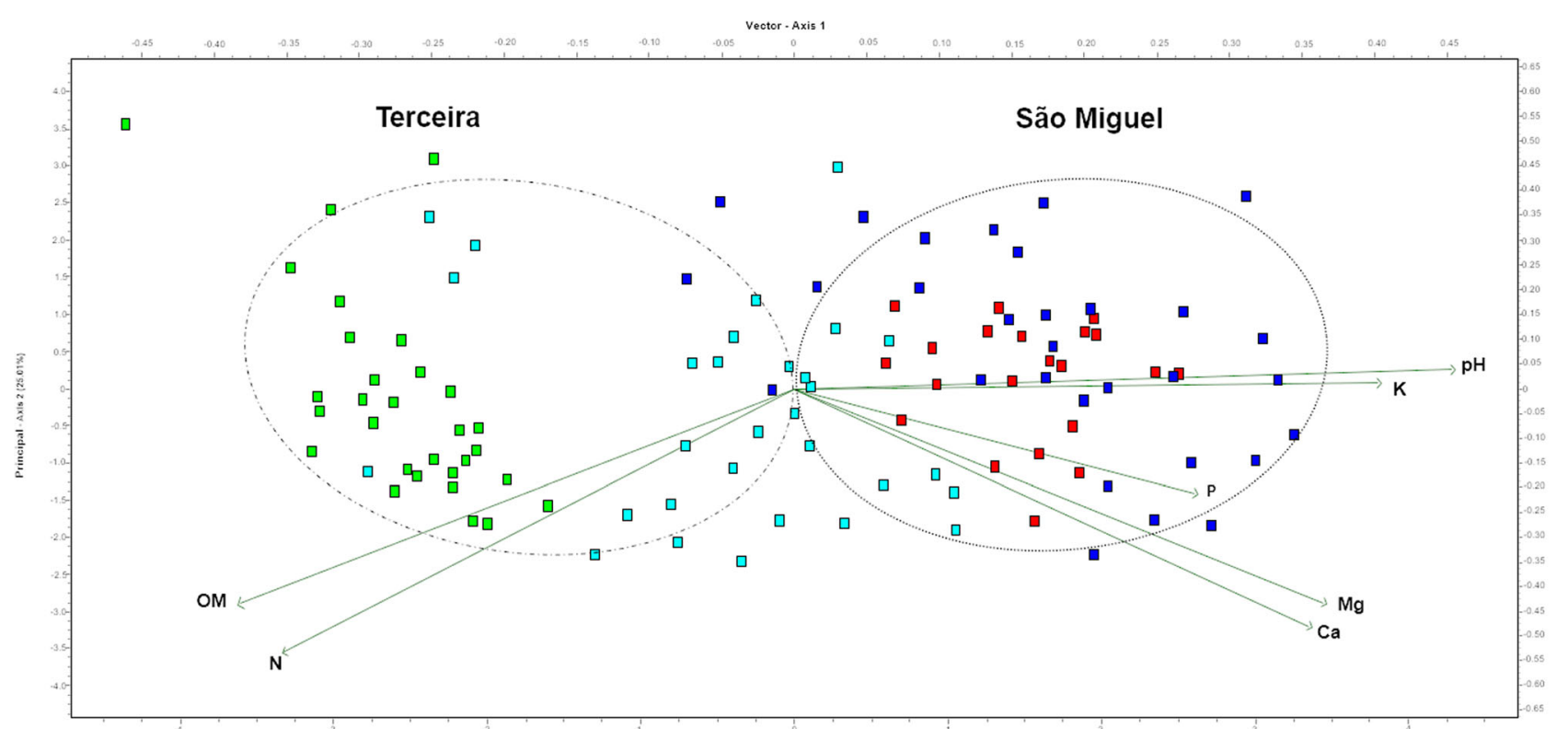

Fig. 1 Score plot for the PCA of soil properties for samples from the four native fragments studied (TB green squares, TS blue squares, SG - red squares and SR - dark blue squares). The two axes explained $78 \%$ of the variation present in the samples 
Table 2 Occurrence of glomeromycotan species in Terceira (Terra Brava - TB and Serreta - TS) and S. Miguel islands (Lombo Gordo - SG and Ribeira Quente SR) during the three sampling dates Autumn of $2012\left(\mathrm{Au}^{1}\right)$, Spring of $2013(\mathrm{SP})$ and Autumn of $2013\left(\mathrm{Au}^{2}\right)$

\begin{tabular}{|c|c|c|c|c|c|c|c|c|c|c|c|c|}
\hline \multirow[t]{3}{*}{ Taxa recorded } & \multicolumn{6}{|c|}{ Terceira } & \multicolumn{6}{|c|}{ São Miguel } \\
\hline & \multicolumn{3}{|l|}{ TB } & \multicolumn{3}{|l|}{ TS } & \multicolumn{3}{|l|}{ SG } & \multicolumn{3}{|l|}{ SR } \\
\hline & $\mathrm{Au}^{(1)}$ & SP & $\mathrm{Au}^{(2)}$ & $\mathrm{Au}^{(1)}$ & SP & $\mathrm{Au}^{(2)}$ & $\mathrm{Au}^{(1)}$ & SP & $\mathrm{Au}^{(2)}$ & $\mathrm{Au}^{(1)}$ & SP & $\mathrm{Au}^{(2)}$ \\
\hline Acaulospora brasiliensis & $\mathrm{x}$ & $\mathrm{x}$ & $\mathrm{x}$ & $\mathrm{x}$ & $\mathrm{x}$ & $\mathrm{x}$ & & & & & & \\
\hline Acaulospora cavernata & $\mathrm{x}$ & $\mathrm{x}$ & $\mathrm{x}$ & & & & & $\mathrm{x}$ & & & & \\
\hline Acaulospora delicata & $\mathrm{x}$ & & & $\mathrm{x}$ & & & & & & & & \\
\hline Acaulospora elegans & $\mathrm{x}$ & $\mathrm{x}$ & & & & & & & & & & \\
\hline Acaulospora cf. koskei & $\mathrm{x}$ & & $\mathrm{x}$ & & & & & & & & & \\
\hline Acaulospora lacunosa & $\mathrm{x}$ & $\mathrm{x}$ & $\mathrm{x}$ & $\mathrm{x}$ & $\mathrm{x}$ & $\mathrm{x}$ & & & & & & \\
\hline Acaulospora laevis & & & & & $\mathrm{x}$ & $\mathrm{x}$ & $\mathrm{x}$ & & & & & \\
\hline Acaulospora mellea & & & $\mathrm{x}$ & $\mathrm{x}$ & $\mathrm{x}$ & $\mathrm{x}$ & $\mathrm{x}$ & $\mathrm{x}$ & $\mathrm{x}$ & $\mathrm{x}$ & $\mathrm{x}$ & $\mathrm{x}$ \\
\hline Acaulospora spinosa & & & & $\mathrm{x}$ & $\mathrm{x}$ & $\mathrm{x}$ & & & & & & \\
\hline Acaulospora sp.1 & & $\mathrm{x}$ & $\mathrm{x}$ & & $\mathrm{x}$ & & & & & & & \\
\hline Acaulospora sp.2 & & & $\mathrm{x}$ & & & & & & & & & \\
\hline Acaulospora sp. 3 & & $\mathrm{x}$ & $\mathrm{x}$ & & & & & & & & & \\
\hline Acaulospora sp.4 & & & & & & $\mathrm{x}$ & & & & & & \\
\hline Acaulospora sp. 5 & & $\mathrm{x}$ & & & & $\mathrm{x}$ & & & & & & \\
\hline Ambispora appendicula & $\mathrm{x}$ & & & & $\mathrm{x}$ & $\mathrm{x}$ & & & & & & \\
\hline Ambispora fennica & & & & & $\mathrm{x}$ & & $\mathrm{x}$ & & & & $\mathrm{x}$ & \\
\hline Archaeospora trappei & & & & & & $\mathrm{x}$ & $\mathrm{x}$ & $\mathrm{x}$ & $\mathrm{x}$ & $\mathrm{x}$ & $\mathrm{x}$ & $\mathrm{x}$ \\
\hline Archaeospora sp. & & $\mathrm{x}$ & $\mathrm{x}$ & & $\mathrm{x}$ & $\mathrm{x}$ & & & & & & \\
\hline Claroideoglomus claroideum & $\mathrm{x}$ & $\mathrm{x}$ & & $\mathrm{x}$ & & & $\mathrm{x}$ & & $\mathrm{x}$ & $\mathrm{x}$ & $\mathrm{x}$ & $\mathrm{x}$ \\
\hline Diversispora celata & & $\mathrm{x}$ & & & & & & & & & $\mathrm{x}$ & \\
\hline Diversispora sp.1 & & $\mathrm{x}$ & $\mathrm{x}$ & & $\mathrm{x}$ & & & $\mathrm{x}$ & $\mathrm{x}$ & & $\mathrm{x}$ & $\mathrm{x}$ \\
\hline Diversispora sp.2 & & & $\mathrm{x}$ & & & & $\mathrm{x}$ & $\mathrm{x}$ & $\mathrm{x}$ & & $\mathrm{x}$ & $\mathrm{x}$ \\
\hline Gigaspora margarita & $\mathrm{x}$ & $\mathrm{x}$ & & $\mathrm{x}$ & $\mathrm{x}$ & $\mathrm{x}$ & $\mathrm{x}$ & $\mathrm{x}$ & $\mathrm{x}$ & $\mathrm{x}$ & $\mathrm{x}$ & $\mathrm{x}$ \\
\hline Glomus albidum & & & & & & & & $\mathrm{x}$ & & & $\mathrm{x}$ & \\
\hline Glomus sp.1 & $\mathrm{x}$ & & & $\mathrm{x}$ & & & $\mathrm{x}$ & $\mathrm{x}$ & $\mathrm{x}$ & $\mathrm{x}$ & $\mathrm{x}$ & $\mathrm{x}$ \\
\hline Glomus sp.2 & & & $\mathrm{x}$ & $\mathrm{x}$ & $\mathrm{x}$ & $\mathrm{x}$ & $\mathrm{x}$ & $\mathrm{x}$ & $\mathrm{x}$ & $\mathrm{x}$ & $\mathrm{x}$ & $\mathrm{x}$ \\
\hline Glomeraceae sp.1 & $\mathrm{x}$ & $\mathrm{x}$ & $\mathrm{x}$ & $\mathrm{x}$ & $\mathrm{x}$ & $\mathrm{x}$ & $\mathrm{x}$ & $\mathrm{x}$ & $\mathrm{x}$ & $\mathrm{x}$ & $\mathrm{x}$ & $\mathrm{x}$ \\
\hline Glomoid spore type 1 & & $\mathrm{x}$ & $\mathrm{x}$ & & $\mathrm{x}$ & & & $\mathrm{x}$ & & & $\mathrm{x}$ & \\
\hline Glomoid spore type 2 & & & & & & & & $\mathrm{x}$ & & & & \\
\hline Glomoid spore type 3 & & $\mathrm{x}$ & $\mathrm{x}$ & & & & & & & & & \\
\hline Glomoid spore type 4 & $\mathrm{x}$ & $\mathrm{x}$ & $\mathrm{x}$ & $\mathrm{x}$ & $\mathrm{x}$ & $\mathrm{x}$ & & & & & & \\
\hline Glomoid spore type 5 & & $\mathrm{x}$ & $\mathrm{x}$ & & $\mathrm{x}$ & & & & $\mathrm{x}$ & & $\mathrm{x}$ & \\
\hline Glomoid spore type 6 & $\mathrm{x}$ & $\mathrm{x}$ & $\mathrm{x}$ & $\mathrm{x}$ & $\mathrm{x}$ & $\mathrm{x}$ & $\mathrm{x}$ & & & & $\mathrm{x}$ & $\mathrm{x}$ \\
\hline Glomoid spore type 7 & & $\mathrm{x}$ & $\mathrm{x}$ & & & & & & & & & \\
\hline Glomoid spore type 8 & & & & & & $\mathrm{x}$ & & $\mathrm{x}$ & & & & \\
\hline Glomoid spore type 9 & & $\mathrm{x}$ & & & & & & & & & & \\
\hline Glomoid spore type 10 & & & & & & & & & & & $\mathrm{x}$ & \\
\hline Pacispora sp. & & $\mathrm{x}$ & & & & & & & & & $\mathrm{x}$ & \\
\hline Paraglomus sp. & & & & & & & & & & & & \\
\hline Rhizophagus clarus & $\mathrm{x}$ & $\mathrm{x}$ & $\mathrm{x}$ & & $\mathrm{x}$ & & & & & & & \\
\hline Rhizophagus sp.1 & & & $\mathrm{x}$ & & & & & & & & & \\
\hline Rhizophagus sp.2 & & $\mathrm{x}$ & & & & & & & & & & \\
\hline Sclerocystis rubiformis & & $\mathrm{x}$ & $\mathrm{x}$ & & $\mathrm{x}$ & $\mathrm{x}$ & & & & & $\mathrm{x}$ & $\mathrm{x}$ \\
\hline Scutellospora sp. & & $\mathrm{x}$ & & & & $\mathrm{x}$ & & $\mathrm{x}$ & $\mathrm{x}$ & & $\mathrm{x}$ & $\mathrm{x}$ \\
\hline Septoglomus constrictum & & $\mathrm{x}$ & & & $\mathrm{x}$ & $\mathrm{x}$ & & $\mathrm{x}$ & $\mathrm{x}$ & & $\mathrm{x}$ & \\
\hline
\end{tabular}

the maximum spore density in Spring with an average of 462 spores per $50 \mathrm{~cm}^{3}$ (Fig. 2b).

Spores of species in the Acaulosporaceae was significantly higher in Terceira than in São Miguel (Kruskal-Wallis test; Acaulosporaceae spore density: $H=55.29 ; p<0.001$ ) (Fig. $3 \mathrm{a})$ and among the four native fragments (Kruskal-Wallis test; Acaulosporaceae spore density: $H=45.48 ; p<0.001$ ) (Fig. $3 b)$, but no significant differences were found among the sampling dates. TB showed the highest spore density from the
Acaulosporaceae and SG the lowest, but no significant differences were observed between TS and SR. Spore density of members of Diversisporaceae also varied significantly between the two islands (Kruskal-Wallis test; Diversisporaceae spore density: $H=19.16 ; p<0.001$ ) (Fig. 3c), but, on the contrary, density of species from this family was higher in São Miguel than in Terceira. Significant differences were found in Diversiporaceae spore density among fragments (Kruskal-Wallis test; Diversisporaceae spore density: $H=$ 
Table 3 Species and unidentified morpho-taxa of glomeromycotan spores extracted from native forests of Terceira (Terra Brava - TB and Serreta - TS) and São Miguel (Lombo -SG and Ribeira Quente - SR).
Relative glomeromycotan spore density (RD), frequency of occurrence (FR) and importance value (IV) of glomeromycotan spores identified from 120 soil samples

\begin{tabular}{|c|c|c|c|c|c|c|c|c|c|c|c|c|c|}
\hline \multirow[t]{3}{*}{ Species recorded } & \multirow{3}{*}{$\begin{array}{l}\text { Accession number to } \\
\text { GenBank (Melo et al. 2018) } \\
\text { Number }\end{array}$} & \multicolumn{6}{|c|}{ Terceira } & \multicolumn{6}{|c|}{ São Miguel } \\
\hline & & \multicolumn{3}{|l|}{ TB } & \multicolumn{3}{|l|}{$\mathrm{TS}$} & \multicolumn{3}{|l|}{ SG } & \multicolumn{3}{|l|}{ SR } \\
\hline & & $\mathrm{RD} \%$ & FR \% & IV \% & $\mathrm{RD} \%$ & FR \% & IV \% & $\mathrm{RD} \%$ & FR \% & IV \% & $\mathrm{RD} \%$ & FR \% & IV \% \\
\hline Acaulospora brasiliensis & КР756414-КР756420 & 11.84 & 93.33 & 52.59 & 1.94 & 63.33 & 32.62 & 3.15 & 9.52 & 6.34 & & & \\
\hline Acaulospora cavernata & КР756472-КР756473 & 1.60 & 2.00 & 1.80 & & & & & & & & & \\
\hline Acaulospora delicata & & 0.25 & 3.33 & 1.68 & 0.13 & 3.33 & 1.67 & & & & & & \\
\hline Acaulospora elegans & & 0.50 & 6.67 & 3.36 & & & & & & & & & \\
\hline Acaulospora cf. koskei & KP756421-KP756426 & 0.14 & 6.67 & 3.42 & & & & & & & & & \\
\hline Acaulospora lacunosa & КР756427-КР756436 & 6.81 & 76.67 & 41.74 & 2.61 & 56.67 & 29.64 & & & & & & \\
\hline Acaulospora laevis & КР756437-КР756447 & 0.87 & 6.00 & 3.44 & 0.83 & 1.00 & 5.41 & 0.26 & 2.51 & 0.13 & & & \\
\hline Acaulospora mellea & KP756464-KP756471 & 0.87 & 6.67 & 3.38 & 0.18 & 13.33 & 6.75 & 5.31 & 33.33 & 19.32 & 17.85 & 9.00 & 53.53 \\
\hline Acaulospora spinosa & & & & & 0.83 & 1.00 & 5.41 & & & & & & \\
\hline Acaulospora sp. 1 & KP756448-KP756452 & 3.46 & 13.33 & 8.39 & 0.52 & 16.67 & 8.36 & & & & & & \\
\hline Acaulospora sp. 2 & & 2.66 & 3.33 & 3.00 & & & & & & & & & \\
\hline Acaulospora sp. 3 & & 0.90 & 1.00 & 5.45 & & & & & & & & & \\
\hline Acaulospora sp. 4 & & & & & 0.27 & 3.33 & 1.68 & & & & & & \\
\hline Acaulospora sp. 5 & & 0.37 & 3.33 & 1.69 & 0.13 & 3.33 & 1.67 & & & & & & \\
\hline Ambispora appendicula & & 0.62 & 6.67 & 3.36 & 0.62 & 6.67 & 3.36 & & & & & & \\
\hline Ambispora fennica & & & & & 0.13 & 3.33 & 1.67 & 0.15 & 4.76 & 2.43 & 0.29 & 6.67 & 3.48 \\
\hline Archaeospora trappei & & & & & 0.41 & 3.33 & 1.69 & 0.74 & 19.48 & 9.89 & 1.48 & 16.67 & 8.86 \\
\hline Archaeospora sp. & КР756477-КР756481 & 0.47 & 13.33 & 6.90 & 0.24 & 16.67 & 8.45 & & & & & & \\
\hline $\begin{array}{l}\text { Claroideoglomus } \\
\text { claroideum }\end{array}$ & KP756498-KР756499 & 0.87 & 6.67 & 3.38 & 0.12 & 6.67 & 3.40 & 1.58 & 23.90 & 12.69 & 0.89 & 23.33 & 12.11 \\
\hline Diversispora celata & & 0.12 & 3.33 & 1.67 & & & & & & & 0.16 & 3.33 & 1.75 \\
\hline Diversispora sp.1 & КР756537-КР756538 & 4.12 & 5.00 & 27.59 & 0.12 & 3.33 & 1.73 & 14.62 & 57.14 & 35.88 & 6.17 & 46.67 & 26.41 \\
\hline Diversispora sp.2 & КР756474-КР756476 & 0.12 & 3.33 & 1.67 & & & & 1.52 & 38.95 & 19.57 & 0.44 & 13.33 & 6.89 \\
\hline Gigaspora margarita & КР756500-КР756515 & 0.24 & 13.33 & 6.79 & 1.38 & 53.33 & 27.36 & 1.68 & 52.39 & 27.32 & 12.83 & 76.67 & 44.75 \\
\hline Glomus albidum & & & & & & & & 0.16 & 4.76 & 2.46 & 0.95 & 3.33 & 1.71 \\
\hline Glomus sp.1 & КР756533-КР756534 & 0.25 & 3.33 & 1.68 & 0.12 & 6.67 & 3.40 & 1.52 & 33.33 & 21.92 & 3.24 & 13.33 & 8.29 \\
\hline Glomus sp.2 & KP756522-KР756532 & 1.43 & 16.67 & 9.58 & 35.59 & 63.33 & 49.42 & 9.67 & 71.43 & 4.55 & 26.77 & 8.00 & 53.39 \\
\hline Glomeraceae sp.1 & KP756482-KP756497 & 3.69 & 46.67 & 25.18 & 45.15 & 83.33 & 64.24 & 44.32 & 66.67 & 55.49 & 22.90 & 63.33 & 43.11 \\
\hline Glomoid spore type 1 & & 0.31 & 23.33 & 11.82 & 0.13 & 16.67 & 8.38 & 1.74 & 4.76 & 3.25 & 1.48 & 3.33 & 2.20 \\
\hline Glomoid spore type 2 & & & & & & & & 1.14 & 4.76 & 2.93 & & & \\
\hline Glomoid spore type 3 & & 0.87 & 6.67 & 3.38 & & & & & & & & & \\
\hline Glomoid spore type 4 & & 6.94 & 5.00 & 28.47 & 0.22 & 2.00 & 1.18 & & & & & & \\
\hline Glomoid spore type 5 & & 1.57 & 26.67 & 14.12 & 0.13 & 6.67 & 3.45 & 0.37 & 4.76 & 2.56 & 0.32 & 3.33 & 1.68 \\
\hline Glomoid spore type 6 & & 45.34 & 73.33 & 59.18 & 9.46 & 63.33 & 36.39 & 0.43 & 4.76 & 2.59 & 2.96 & 2.00 & 11.48 \\
\hline Glomoid spore type 7 & & 0.22 & 1.00 & 5.11 & & & & & & & & & \\
\hline Glomoid spore type 8 & & & & & 0.01 & 3.33 & 1.67 & 0.47 & 4.76 & 2.62 & & & \\
\hline Glomoid spore type 9 & & 1.52 & 3.33 & 2.43 & & & & & & & & & \\
\hline Glomoid spore type 10 & & & & & & & & & & & 0.70 & 3.33 & 2.16 \\
\hline Pacispora sp. & & 0.62 & 3.33 & 1.70 & & & & & & & & & \\
\hline Paraglomus sp. & & & & & & & & & & & 1.11 & 3.33 & 2.22 \\
\hline Rhizophagus clarus & & 1.42 & 66.67 & 34.45 & 0.93 & 3.33 & 1.71 & & & & & & \\
\hline Rhizophagus sp.1 & KP756535-КР756536 & 0.14 & 6.67 & 3.42 & & & & & & & & & \\
\hline Rhizophagus sp.2 & & 2.17 & 3.33 & 2.75 & & & & & & & & & \\
\hline
\end{tabular}


Table 3 (continued)

\begin{tabular}{|c|c|c|c|c|c|c|c|c|c|c|c|c|c|}
\hline \multirow[t]{3}{*}{ Species recorded } & \multirow{3}{*}{$\begin{array}{l}\text { Accession number to } \\
\text { GenBank (Melo et al. 2018) } \\
\text { Number }\end{array}$} & \multicolumn{6}{|c|}{ Terceira } & \multicolumn{6}{|c|}{ São Miguel } \\
\hline & & \multicolumn{3}{|l|}{ TB } & \multicolumn{3}{|l|}{$\mathrm{TS}$} & \multicolumn{3}{|l|}{ SG } & \multicolumn{3}{|l|}{ SR } \\
\hline & & $\mathrm{RD} \%$ & FR \% & IV \% & $\mathrm{RD} \%$ & FR $\%$ & IV \% & $\mathrm{RD} \%$ & FR $\%$ & IV \% & $\mathrm{RD} \%$ & FR \% & IV \% \\
\hline Sclerocystis rubiformis & & 1.58 & 23.33 & 12.46 & 1.15 & 1.00 & 5.57 & & & & & & \\
\hline Scutellospora sp. & KP756516-KР756521 & 0.50 & 3.33 & 1.69 & 0.27 & 3.33 & 1.68 & 0.26 & 14.29 & 7.27 & 0.48 & 13.33 & 6.95 \\
\hline Septoglomus constrictum & & 0.22 & 13.33 & 6.78 & 0.30 & 1.00 & 5.15 & 2.37 & 33.33 & 17.85 & 2.64 & 2.00 & 11.32 \\
\hline
\end{tabular}

24.54; $p<0.001$ ) (Fig. 3d) and among sampling dates (Kruskal-Wallis test; Diversisporaceae spore density: $H=$ 33.33; $p<0.001$ ) (Fig. 3e). Spore density difference between TS (lowest) and SG (highest) was significant, but SG, SR, or TB did not differ, and over time was its minimum during the first autumn and its maximum at the second autumn.

A similar pattern was observed for spore density from the Gigasporaceae which varied significantly between the two islands (Kruskal-Wallis test; Gigasporaceae spore density: $H$ $=12.17 ; p<0.001$ ) (Fig. 3f) and among the four native fragments (Kruskal-Wallis test; Gigasporaceae spore density: $H=$ 33.88; $p<0.001$ ) (Fig. 3g), but no significant difference was observed among sampling dates. Density of members of the Gigasporaceae was higher in São Miguel than in Terceira especially in SR, and lowest in TB.

Spore density of members of the Glomeraceae differed significantly between Terceira and São Miguel (KruskalWallis test; Glomeraceae spore density: $H=25.75$; $p<$ 0.001 ) (Fig. 3h) among native fragments (Kruskal-Wallis test; Glomeraceae spore density: $H=30.36$; $p<0.001$ ) (Fig. 3i) and sampling dates (Kruskal-Wallis test; Glomeraceae spore density: $H=26.55$; $p<0.001$ ) (Fig. 3j). Such spore density was higher in Terceira than in São Miguel, with TS being highest, followed by TB, and SG lowest, but no significant difference was found between the two sites from São Miguel. Over the time, spore density from Glomeraceae peaked at the spring, although no significant difference was found between this season and the second autumn, and achieved its minimum on the first autumn.

\section{Mycorrhizal colonisation}

All the $P$. azorica samples were mycorrhizal, with colonisation ranging in Terceira from 77 to $97 \%$ (mean 87\%) and in São Miguel from 57 to $86 \%$ (mean $76 \%$ ). However, samples from Terceira showed significant higher colonisation than samples from São Miguel (Kruskal-Wallis test; total mycorrhizal colonisation: $H=54.64 ; p<0.001$ ) (Fig. 4a), due to the lower level of colonisation at both native fragments in this island (Kruskal-Wallis test; total mycorrhizal colonisation: $H$ $=55.29 ; p<0.001$ ) particularly in the SG samples (Fig. 4b).
Mycorrhizal colonisation also showed a clear temporal pattern among sampling dates (Kruskal-Wallis test; total mycorrhizal colonisation: $H=26.84$; $p<0.001)$. Overall, average mycorrhizal colonisation was highest during spring and lowest in the second autumn (Fig. 4c).

\section{Colonisation type of mycorrhiza}

In all sites, AM fungal colonisation byarbuscules were the most common form of colonisation found in the roots of $P$. azorica samples but varied significantly between island (Kruskal-Wallis test; arbuscular colonisation: $H=50.93 ; p<$ 0.001 ), among native fragments (Kruskal-Wallis test; arbuscular colonisation: $H=51.03 ; p<0.001)$ and sampling dates (Kruskal-Wallis test; arbuscular colonisation: $H=43.19$; $p<0.001)$. Arbuscular colonisation was higher in Terceira than in São Miguel especially in TB during the spring, although no significant difference occurred between the two native fragments from Terceira. The same pattern was observed to hyphal colonisation, i.e., significant variation between the two islands (Kruskal-Wallis test; hyphal colonisation: $H=14.08 ; p<0.001$ ), among native fragments (KruskalWallis test; hyphal colonisation: $H=14.27 ; p=0.003)$ and sampling dates (Kruskal-Wallis test; hyphal colonisation: $H=$ 27.74; $p<0.001$ ). Hyphal colonisation was highest in Terceira than in São Miguel, particularly in TS, although no significant difference was observed between the two native fragments from Terceira, and lowest in SG. Hyphal colonisation was significantly higher in the second autumn than in the spring and the first autumn. Numbers of intraradical spores, or vesicles, also changed significantly between Terceira and São Miguel (Kruskal-Wallis test; vesicular colonisation: $H=$ $16.61 ; p<0.001)$ and among native fragments $(F=18.02 ; p$ $<0.001)$, but no significant difference was observed in these characters among sampling dates.

\section{Effect of environmental variables on spore density}

The four forest fragments of $P$. azorica differed significantly in most of the soil and climatic factors evaluated (Table 1), thus revealing the relationships between the environmental 
Fig. 2 a) AMF spore density (mean $\pm \mathrm{SE}, N=90$ ) per $50 \mathrm{~g}$ of soil in each island (Terceira and São Miguel) and b) AMF spore density (mean $\pm \mathrm{SE}, N=10$ ) by sampling time (September 2012, $\mathrm{AU}^{(1)}$; May 2013, SP; September 2013, $\left.\mathrm{AU}^{(2)}\right)$ within each fragment (Terra Brava - TB; Serreta - TS; Lombo Gordo - SG; Ribeira Quente - SR). Different letters above each bar indicate significant differences in AMF spore density between fragments or sampling date (Tukey test, $p<$ 0.05 ) a)

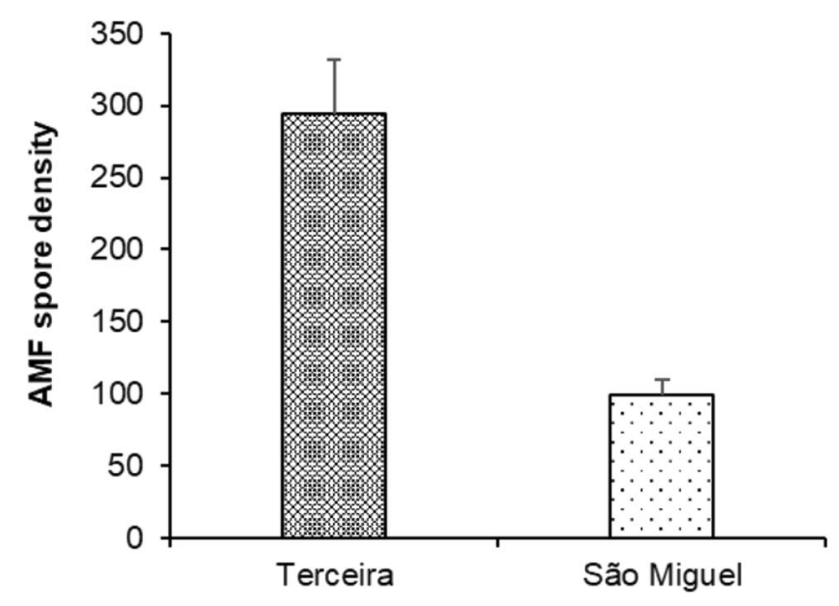

b)

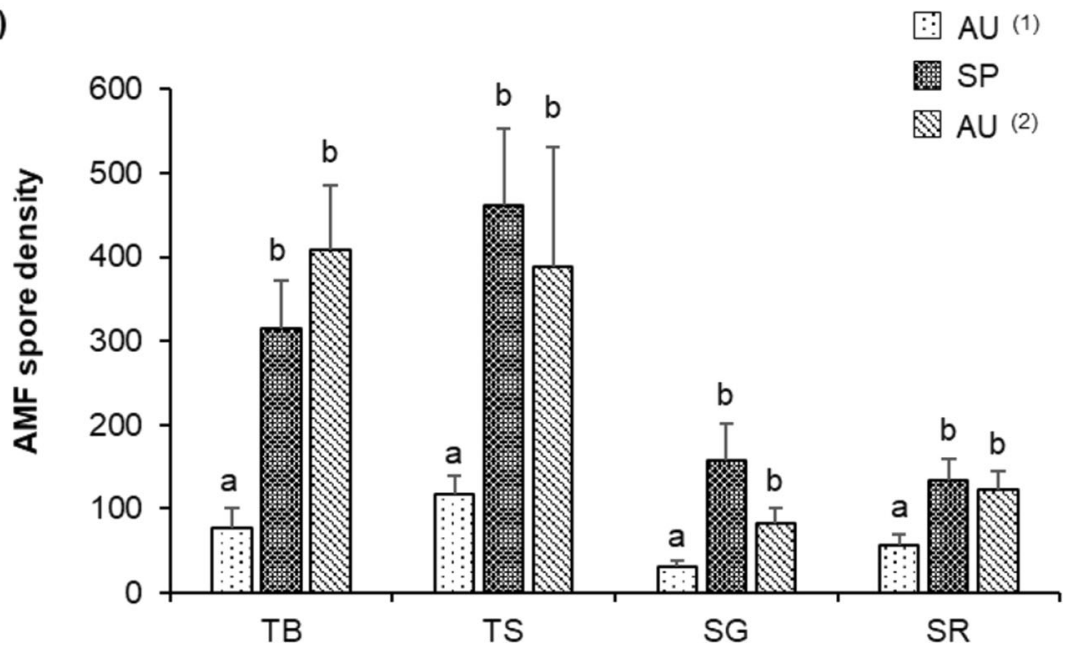

factors and AMF communities. The effect of differences in soil properties and climatic variables on spore density varied depending on AMF family.

From the regression analysis, the best model for the effect of environmental variables on Acaulosporaceae spore density included $\mathrm{pH}(t=-2.73, p=0.007)$, relative air humidity $(t=$ 2.62, $p=0.01)$ and soil available $P(t=-2.19, p=0.03)$ (Table 4). Taxa from the Acaulosporaceae were negatively correlated with soil $\mathrm{pH}$ and available $P$, while the relative air humidity influenced positively the sporulation of species in this family. The best model for gigasporaceous spores shows an increase with soil available $\mathrm{K}(t=5.08, p<0.001)$, and a decrease with relative air humidity $(t=-3.65, p<0.001)$ and soil available $\operatorname{Mg}(t=-2.80, p=0.006)$ (Table 4).

In comparison, multiple regression analysis of Glomeraceae family members revealed relationships involving all the climatic variables and soil properties, except organic matter and available soil P. The spore density from the Glomeraceae showed a strong positive correlation with monthly rainfall $(t=3.83, p<0.001)$ and available soil magnesium $(t=2.92, p=0.004)$, but a strong negative correlation with mean monthly temperature $(t=-3.65, p<0.001)$, relative air humidity $(t=-3.60, p<0.001)$, available soil $\mathrm{K}(t$ $=-3.09, p=0.003)$ and $\mathrm{pH}(t=-2.66, p=0.009)$ (Table 4). The best model for the effect of environmental variables on spore density of taxa in the Diversisporaceae included monthly rainfall $(t=6.79, p<0.001)$, mean monthly temperature $(t=$ $-5.73, p<0.001)$ and soil available $\mathrm{K}(t=3.87, p<0.001)$ (Table 4). Density of spores from the Diversisporaceae was positively influenced by monthly rain and available soil $\mathrm{K}$, but negatively affected by the increasing of mean monthly temperature.

\section{Effect of environmental variables on mycorrhizal colonisation}

Mycorrhizal colonisation (all measurements) was correlated with environmental variables. The best model for the effect of environmental variables on total colonisation included monthly rainfall $(t=-5.97, p<0.001), \mathrm{pH}(t=-4.57, p<$ 
Island
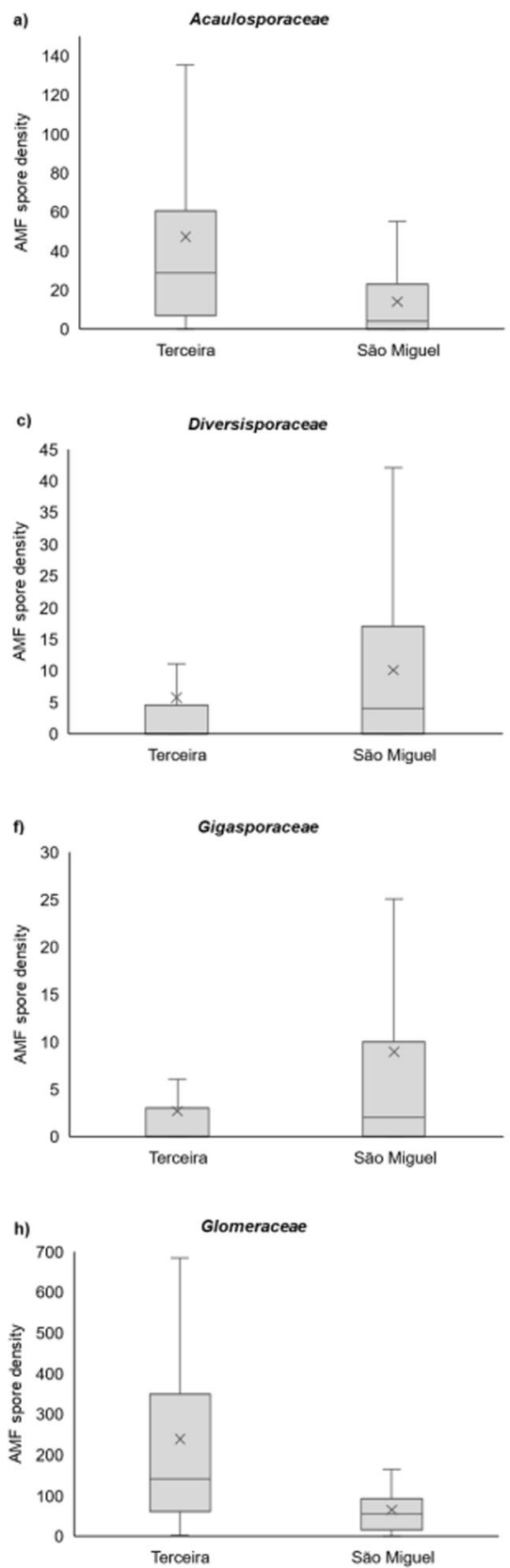

Site

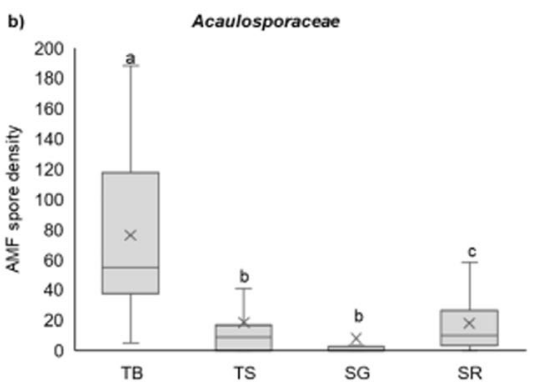

d)

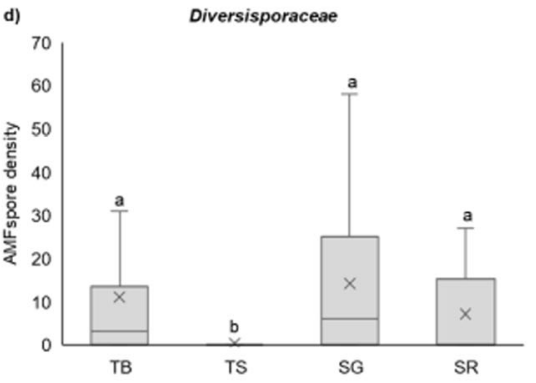

g) Gigasporaceae
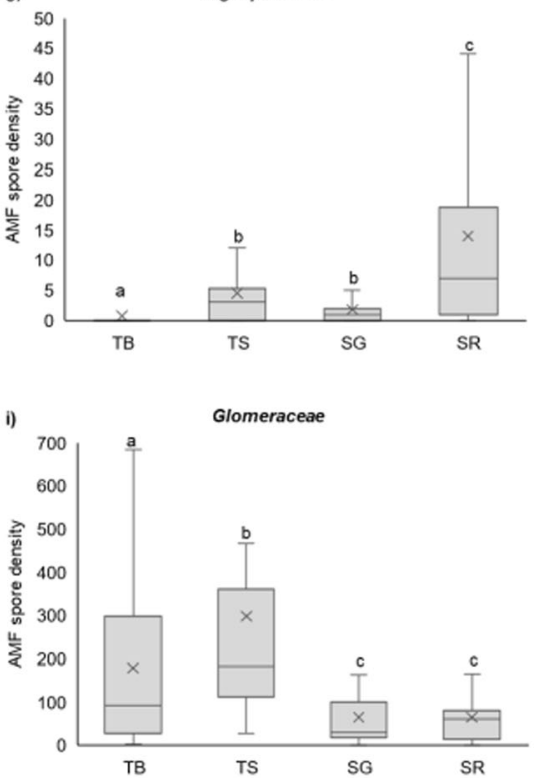

Sampling date
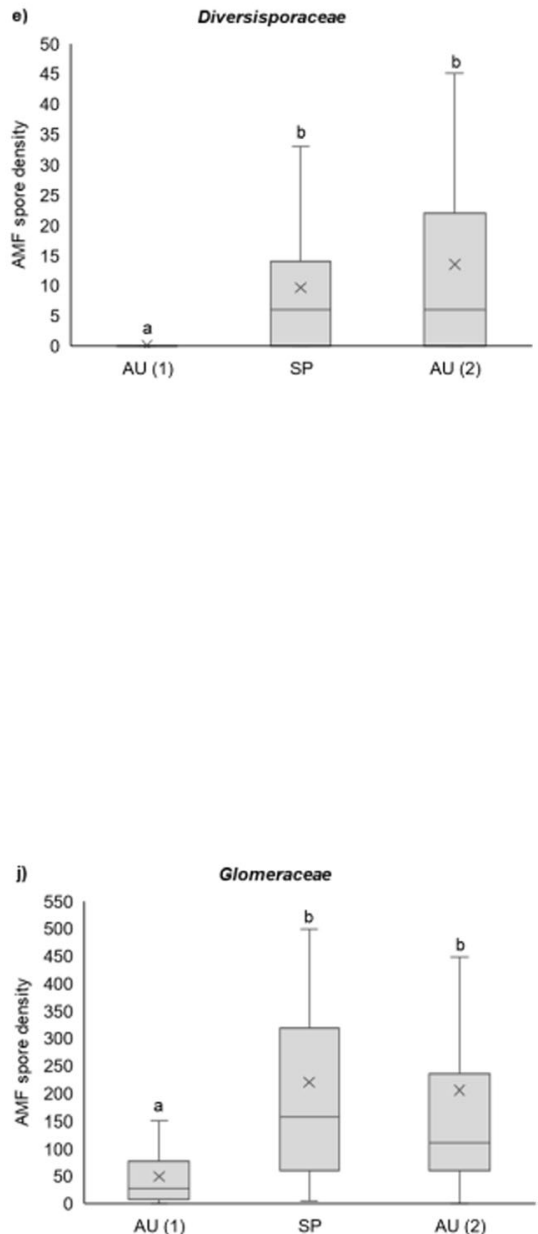

Fig. 3 Spore density of each family of Glomeromycota in each island and in the four forest fragments (Terra Brava, TB; Serreta, TS; Lombo Gordo, SG; Ribeira Quente, SR) for members of the families: a-b) Acaulosporaceae; c-e) Diversisporaceaeae; f-g) Gigasporaceae; hj) Glomeraceae, obtained during three sampling dates time (September

$0.001)$, available soil $P(t=-4.11, p<0.001)$, available soil $\mathrm{K}$ $(t=-3.53, p<0.001)$ and available soil $\mathrm{Mg}(t=3.22, p=$ 0.002 ) (Table 5). Total mycorrhizal colonisation was negatively affected by the increase in $\mathrm{pH}$, and the available soil $P$ and $\mathrm{K}$, but higher concentrations of available $\mathrm{Mg}$ in the soil seem to improve total mycorrhizal colonisation.

Arbuscular colonisation was correlated with all environmental variables except available soil $\mathrm{Mg}$. The best model
2012, AU(1); May 2013, SP; September 2013, AU(2)). Bold line represents median, $\mathrm{x}$ signals the media and the bottom and top of the box represent lower and upper quartiles. Different letters above each box indicate significant differences in AMF spore density between fragments or sampling date (Kruskal-Wallis test, $p<0.05$ )

for the effect of environmental variables on arbuscular colonisation fits to mean monthly temperature $(t=-9.66, p<$ $0.001)$, monthly rainfall $(t=7.69, p<0.001)$, relative air humidity $(t=-4.73, p<0.001)$, organic matter content $(t=$ 3.57, $p=0.001)$, available soil $P(t=-3.42, p=0.001)$ and available $\mathrm{K}(t=-3.29, p=0.001)$ (Table 5). Equations of regression analysis showed that $68 \%$ of the general variation in arbuscular colonisation could be explained by these 

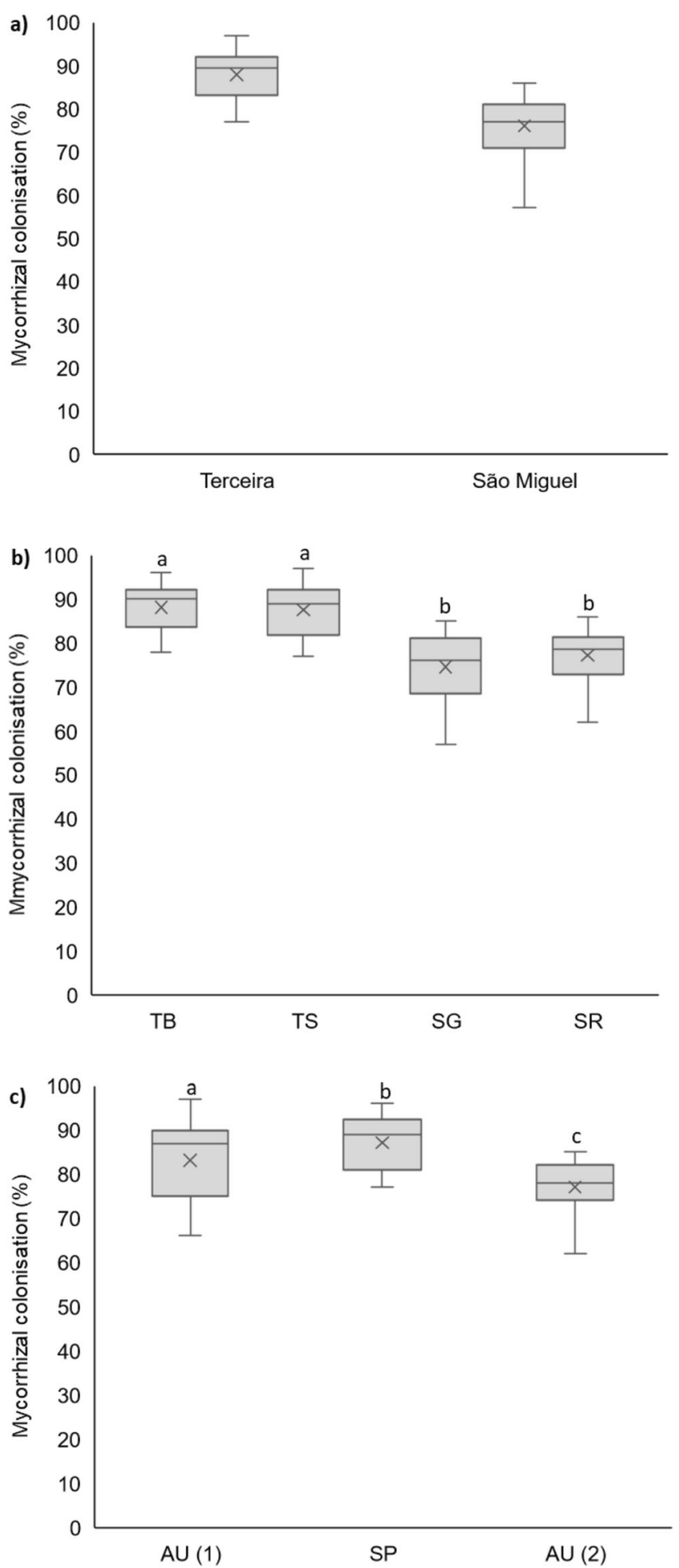

Fig. 4 a) Total mycorrhizal root colonisation in $P$. azorica in each island, b) in the four forest fragments (Terra Brava, TB; Serreta, TS; Lombo Gordo, SG; Ribeira Quente, SR) and c) obtained during three sampling dates (September 2012, AU ${ }^{(1)}$; May 2013, SP; September 2013, AU ${ }^{(2)}$ ). Bold line represents median, $\mathrm{x}$ signals the media and the bottom and top of the box represent lower and upper quartiles. Different letters above each box indicate significant differences in total AMF colonisation between fragments or sampling date (Kruskal-Wallis test, $p<0.05$ ) environmental variables. The best model to intraradical spore or vesicle levels included available soil $\mathrm{K}(t=-3.07, p=$ $0.003)$ and monthly rainfall $(t=-2.53, p=0.01)$ (Table 5$)$, both environmental variables being affecting decrease on vesicular colonisation. By regression analysis, the best model for the effect of environmental variables on hyphal colonisation included monthly rain $(t=6.22, p<0.001)$, mean monthly temperature $(t=4.57, p<0.001)$, available soil $P(t=-3.31, p$ $=0.001)$, organic matter content $(t=2.89, p=0.005)$ and relative air humidity $(t=2.64, p=0.01)$ (Table 5). Hyphal colonisation was positively correlated with all environmental variables but decreased as available soil $P$ increased.

\section{Canonical correspondence analysis}

Canonical correspondence analysis also revealed clear relationships between glomeromycotan community structures and environmental factors (Fig. 5). Figure 5 presents the triplot for the first and second CCA axes, which together explained $71 \%$ of the total variance (Table 6) indicating that environmental factors had significant effects on glomeromycotan spore production in $P$. azorica rhizosphere (Table 6).

Monte Carlo random test indicated that elevation $(F=$ $11.87, p=0.001)$, relative air humidity $(F=3.76, p=$ $0.001)$, mean monthly temperature $(F=2.90, p=0.001)$, soil available $\mathrm{Mg}(F=2.32, p=0.006)$ and soil available $\mathrm{K}(F=$ 2.12, $p=0.002$ ) were the dominating factors affecting spore production in the glomeromycotan communities.

Forty-five glomeromycotan spore morphotypes clustered into four groups (AMF groups I-IV) in the CCA triplots, with different groups responding differently to environment variables (Fig. 5). The spore density of group I, composed mainly of members of Acaulosporaceae, was represented by all TB samples. In this native fragment, the lowest soil available $\mathrm{P}$ and soil $\mathrm{pH}$ were positively correlated to a higher spore density of Acaulosporaceae, as well as to the higher total mycorrhizal colonisation and colonisation by arbuscules and hyphae. Members of this group also showed a positive correlation with elevation, monthly rainfall, relative air humidity, mean monthly temperature and OM content. TB is the $P$. azorica forest fragment located at high elevation, while the remaining three are of low elevation. Moreover, among the four forest fragments, OM content was higher in TB than in the other sites (Table 1). Also, the highest values of monthly rain, relative air humidity and average monthly temperature were also recorded in TB.

The spore density of group II, mainly represented by members of Glomeraceae, was positively related $\mathrm{Mg}$ and $\mathrm{K}$ contents available in soil and $\mathrm{pH}$ and negatively related to elevation, monthly rainfall and mean monthly temperature, suggesting that the members of this family occur preferentially where content of available $\mathrm{P}$ and available $\mathrm{Mg}$ is high (Fig. 5). 
Table 4 Regression equations relating AMF spore density from different AMF families (AMFS) and independent environment variables

\begin{tabular}{llr}
\hline Family & Linear regression & $\mathrm{R}^{2}$ \\
\hline Acaulosporaceae & AMFS $=-4.68 \mathrm{pH}-0.86 \mathrm{P}+4.89 \mathrm{RH}-3.76$ & $0.41^{* * *}$ \\
Diversisporaceae & AMFS $=-0.59 \mathrm{~K}+0.99 \mathrm{MR}-4.42 \mathrm{AMT}+3.21$ & $0.40^{* * *}$ \\
Gigasporaceae & AMFS $=0.75 \mathrm{~K}-0.60 \mathrm{Mg}-4.40 \mathrm{RH}+8.73$ & $0.32^{* * *}$ \\
Glomeraceae & AMFS $=-4.90 \mathrm{pH}-0.64 \mathrm{~K}+0.88 \mathrm{Mg}+0.79 \mathrm{MR}-3.44 \mathrm{AMT}-8.04 \mathrm{RH}+23.10$ & $0.31^{* * *}$ \\
\hline
\end{tabular}

The spore density of group III composed of a mix of species in the Gigasporaceae and Diversisporaceae was positively related with soil available $\mathrm{K}$ and negatively related with the elevation and OM content (Fig. 5). Almost all samples from both sites of São Miguel were present in groups II and III, where soil available $\mathrm{P}$ and $\mathrm{K}$ were significantly higher than in sites from Terceira (Table 1), and where OM content was the lowest.

\section{Discussion}

The roles of AMF have been well documented in different terrestrial ecosystems; however, their ecological role in protected environments, such as natural reserves, is poorly studied. The output of such information has a high socioecological value that is enhanced by the presence of rare or endangered plant species (Triantis et al. 2010), because those niches could constitute vital repositories for the conservation of AMF diversity (Turrini et al. 2010). Overall, a total of 45 AMF spore morphotypes were detected from the four fragments of $P$. azorica forest representing nine families of AMF. This is a high number, as only about 300 species have so-far been classified from spore morphology worldwide for the phylum Glomeromycota (http://amf-phylogeny.com). Almost the same number of AMF spore morphotypes (39) was found in the rhizosphere of Juniperus brevifolia in different fragments of the same native forest, where Acaulosporaceae and Glomeraceae were also the dominant families in rhizosphere of this endemic woody plant (Melo et al. 2017). A study by Velázquez et al. (2013) in a National Park of Argentina also showed that species of Acaulosporaceae were the most widespread and abundant glomeromycotan family with $49 \%$ of spores, followed by
Glomeraceae (40\%) and Gigasporaceae (6\%). As in remnant populations of another endangered tree species (J. brevifolia) spore density among the dominant AMF families, and root colonisation varied significantly between Terceira and São Miguel islands (Melo et al. 2017). This difference between AM fungal communities of Terceira and São Miguel suggests that a regional mosaic of soil types may be important for maintaining high AM fungal diversity (Lekberg et al. 2007; Melo et al. 2017).

Several factors have been identified that may influence AMF distributions, including abiotic (e.g., soil physicochemical properties) and biotic (e.g., host plant) factors, and intrinsic properties of species (e.g., dispersal ability) reviewed by Chaudhary et al. (2008). In this study, significant relationships between AMF spore density of the commonest families, mycorrhizal colonisation, and environmental factors, such as elevation, relative air humidity, soil $\mathrm{pH}$, and soil available $\mathrm{P}$, $\mathrm{K}$, and $\mathrm{Mg}$ were revealed by regression and $\mathrm{CCA}$ analyses. The highest spore density of Acaulosporaceae as well as the highest total mycorrhizal colonisation and colonisation by arbuscules was found in TB, which could be explained by specific environmental factors such as high OM content, rainfall, lower soil $\mathrm{pH}$, and soil available $\mathrm{P}$ and $\mathrm{K}$ that characterise this site. Alguacil et al. (2009) showed that application of $\mathrm{OM}$ can improve soil aggregation and microbial activities, which increased AM fungal diversity in long-term study. In undisturbed environments, Velázquez et al. (2018) also found that the highest organic matter content was positively correlated with the percentage of AMF colonisation.

Variability in water regimes may influence AMF communities within roots by regulating the level of AMF colonisation and the assemblage of different species (Deepika and Kothamasi 2015; Zhang et al. 2016). In Azorean native fragments, relative air humidity and rainfall are significant drivers

Table 5 Regression equations relating AMF root colonisation (\%) and independent environment variables

\begin{tabular}{llll}
\hline Infection type & Linear regression & $\mathrm{R}^{2}$ \\
\hline AMF total colonisation & $\mathrm{AMF}=-1.28 \mathrm{pH}-0.31 \mathrm{P}-0.14 \mathrm{~K}+0.17 \mathrm{Mg}-0.93 \mathrm{AMT}+3.32$ & $0.56^{* * *}$ \\
Arbuscular colonisation & $\mathrm{AMF}=-0.36 \mathrm{P}-0.19 \mathrm{~K}+0.21 \mathrm{OM}+0.42 \mathrm{MR}-2.32 \mathrm{AMT}-2.50 \mathrm{RH}+8.31$ & $0.68^{* * *}$ \\
Vesicular colonisation & $\mathrm{AMF}=-0.10 \mathrm{~K}-0.05 \mathrm{MR}+1.05$ & $0.16^{* *}$ \\
Arbuscular colonisation & $\mathrm{AMF}=-0.36 \mathrm{P}-0.19 \mathrm{~K}+0.21 \mathrm{OM}+0.42 \mathrm{MR}-2.32 \mathrm{AMT}-2.50 \mathrm{RH}+8.31$ & $0.68^{* * *}$ \\
Hyphal colonisation & $\mathrm{AMF}=-0.39 \mathrm{P}+0.18 \mathrm{OM}-0.41 \mathrm{MR}+1.33 \mathrm{AMT}+1.69 \mathrm{RH}-3.52$ & $0.42^{* * *}$ \\
\hline
\end{tabular}




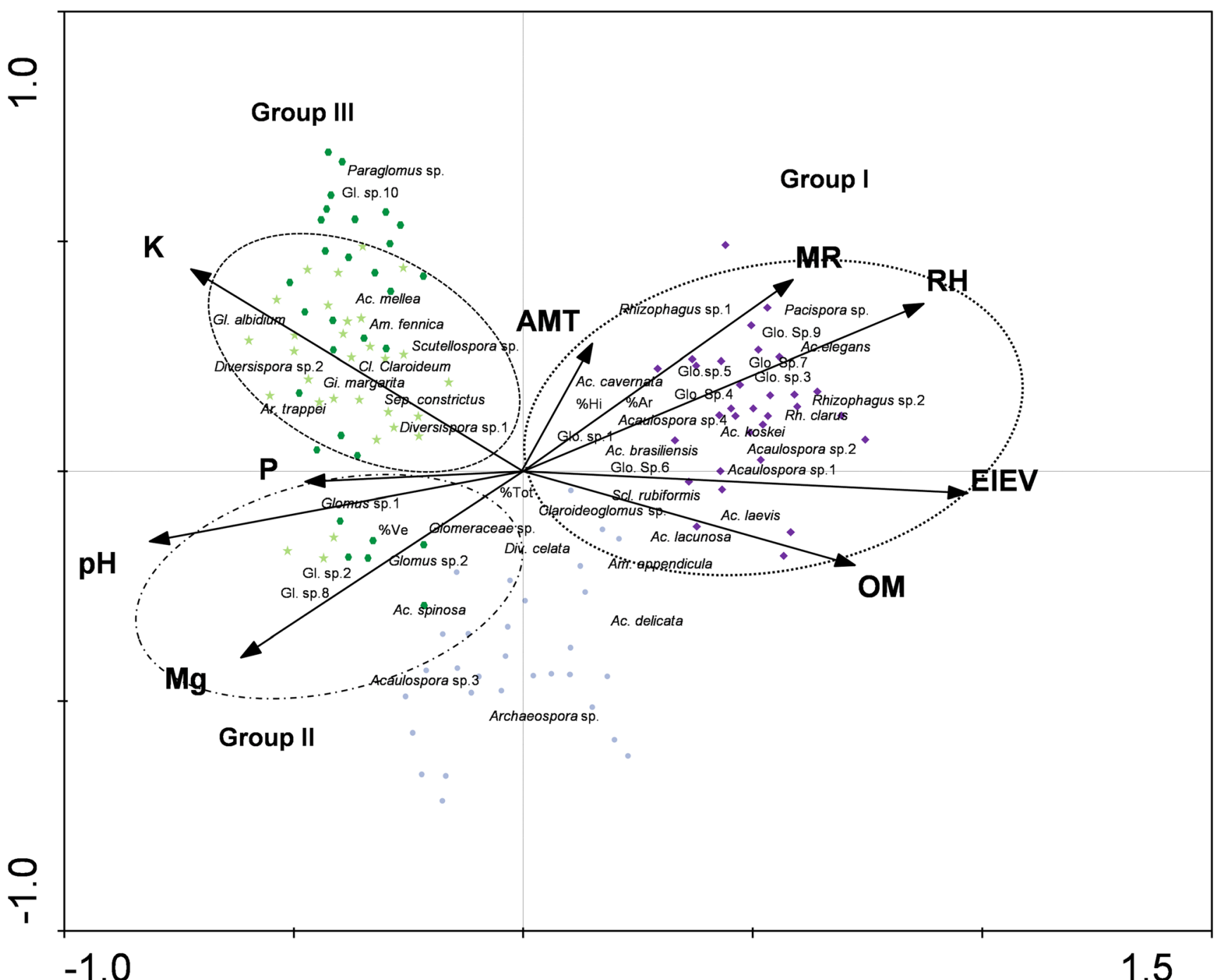

Fig. 5 CCA triplot of glomeromycotan spore density and environment variables in the rhizosphere of $P$. azorica from Terceira (TB - purple diamond and TP - purple circles) and São Miguel (SG - green star and

for AMF spore density, especially for members of the families Acaulosporaceae, Diversisporaceae and Glomeraceae which were positively correlated with these abiotic factors. In contrast, members of the Gigasporaceae showed negative correlation with these climatic variables. Other spore-based studies also showed that rainfall influences AMF sporulation (Sun et al. 2013), spore density and richness (Torrecillas et al. 2013; Melo et al. 2017), and spore community composition (Zhang et al. 2016). Pande and Tarafdar (2004) suggested that increased rainfall and relative air humidity increased AMF colonisation. Zhang et al. (2016) also showed that precipitation directly affected AMF hyphal length density and suggested that water availability could drive the changes of AMF communities at regional scale.

Several studies discussed that Acaulosporaceae members may be confined to the harsh environmental conditions of uplands (Gai et al. 2012; Senés-Guerrero and Schüßler
$\mathrm{SR}$ - green circles). P, Olsen phosphorus; $\mathrm{K}$, potassium; $\mathrm{Mg}$, magnesium; $\mathrm{OM}$, organic matter; $\mathrm{pH}$; ELEV, elevation; MR, monthly rainfall; $\mathrm{RH}$, relative air humidity; AMT, average monthly temperature
2016) and are dominant in protected areas representing more than $50 \%$ of all AMF species found, whilst Glomeraceae represents only $46.3 \%$ (Turrini and Giovannetti 2012; Velázquez et al. 2016). TB is included in Terceira Natural Park, so the relative high root colonisation and AMF spore density in this native fragment may be related to the low disturbance of this ecosystem, as compared to the other studied location at low elevation close to human activities that can influence AMF species composition (Moora et al. 2014; Zhao et al. 2017).

Spore abundance and species richness can vary over elevation gradients ( $\mathrm{Li}$ et al. 2014; Coutinho et al. 2015). In our study, we found a positive correlation of Acaulosporaceae spore density with elevation, which is in contrast with the study of Gai et al. (2012) and Wang et al. (2015) who found a decrease in spore abundance with increasing elevation.

Soil $\mathrm{pH}$ is another environmental factor which can affect AMF (Guo and Gong 2014; Melo et al. 2014, 2017). 
Table 6 CCA ordination of glomeromycotan communities based on spore type and environmental factors

\begin{tabular}{lll}
\hline Analysis results & Axis 1 & Axis 2 \\
\hline Eigen value & 0.50 & 0.21 \\
Correlation coefficient between species and environmental factors & 0.93 & 0.85 \\
Cumulative proportion of species & 9.80 & 14.00 \\
Cumulative proportion of species and environment & 43.90 & 58.80 \\
Total eigen value & 4.97 & 1.22 \\
Total eigen value of CCA & & \\
\hline
\end{tabular}

Mycorrhizal fungi varies in their $\mathrm{pH}$ tolerance; some can be found mainly in low pH soils (e.g., some Acaulosporaceae), whereas others prefer alkaline and neutral substrates (e.g., some Glomeraceae) (Bainard et al. 2014).

Soil phosphorus availability is a critical factor for AMF in terms of both root and soil colonisation, as several studies have indicated that high available $P$ reduced mycorrhizal colonisation and spore production (Lakshmipathy et al. 2012; Gosling et al. 2013). The soil available $P$ in both sites from São Miguel seems to have a significant negative influence on AMF activity. Moreover, members of Glomeraceae were predominantly found in SG and SR where the soil available P is higher than in sites from Terceira (TB; TS) probably showing sporulation adaptability to environmental conditions (Schalamuk and Cabello 2010). These interactions may be attributable to the stimulation by soil phosphorus of spore germination and hyphal growth (Miranda and Harris 1994). The high abundance of Glomeraceae in disturbed habitats has been related to considerable capacity of some of its most frequently found members, e.g., Rhizophagus irregularis, to sporulate (Moora et al. 2014), to colonise roots from already AMF colonised root fragments (Schalamuk and Cabello 2010; Varela-Cervero et al. 2016) and form anastomoses readily (de la Providencia et al. 2013; de Novais et al. 2017). All these characteristics may increase the competitive ability of these fungi, because of the rapidity and extent at which the external mycelium develop in soil.

Soil $\mathrm{K}$ is known to have a stimulatory effect on AMF attributes, by increasing the colonisation rate of AMF under drought stress; usually a minimum of soil $\mathrm{K}$ has been suggested to be a prerequisite for mycorrhizal colonisation in some plant species (Zhang et al. 2017). In our study, $P$. azorica, a negative correlation between soil $\mathrm{K}$ and AMF total root colonisation and colonisation by all AMF structure except hyphal colonisation, was statistically significant. This finding is in agreement with those reported by Panwar et al. (2011) who found a negative correlation between soil $\mathrm{K}$ and AMF sporulation in all the study sites. Conversely, there is a positive correlation between soil available $\mathrm{K}$ and density of spores from members of the Gigasporaceae.

The temporal variation in the spore density (Velázquez et al. 2013; Bouazza et al. 2015; Melo et al. 2017) and in the root colonisation in $P$. azorica probably reflects different strategies among AMF. Several AMF families have seasonal pattern of sporulation, while some show a steady production of spores all year long, sporulation in others occurs at different times in the growing season (Bouamri et al. 2014). Our results showed that sporulation of members of the Diversisporaceae and Glomeraceae fluctuated throughout the three sampling dates, i.e., Diversisporaceae sporulated better during the wet season (autumn), while Glomeraceae sporulated better during warm season (spring). On the contrary, members of the Acaulosporaceae and Gigasporaceae sporulated regardless of the sampling date. This result is opposite to found by Velázquez et al. (2013) who found seasonal patterns on spore abundance of Acaulosporaceae and Gigasporaceae throughout the year. These seasonal differences in sporulation patterns are characteristic of AMF-community dynamics and could be explained by fungal substitutions determined by the differing life strategies of both the fungi and their hosts, adapting to particular climatic conditions, soil moisture regimes and host phenology (Maherali and Klironomos 2007; Powell et al. 2009; Chagnon et al. 2013).

All fungal structure also showed a marked seasonality, particularly in the Spring harvest. The abundance of intraradical structures may be related to host growth, flowering and fruit production (Bainard et al. 2014). Thus, the high percentage of AMF root colonisation in our spring sampling date matches with the start of spring flush of growth of $P$. azorica at the sites, and with the turnover of new short roots which increase the withdrawal of nutrients and starch content in autumn and winter.

During flowering and/or fruiting, the phosphorus demand is high and thus AMF colonisation levels were also high. This can also be demonstrated by the higher percentage of AMF arbuscule colonisation during this season. Likar et al. (2009) also found another peak in arbuscule formation during flowering, in Cruciata laevipes and Mentha piperita and argue that the rate of arbuscule formation can be enhanced during periods of extensive productivity in plants, such as flowering. Arbuscules are formed where nutrients are exchanged between plant and AMF, and a high percentage of arbuscular colonisation indicates an intense activity between the symbiotic partners (Greipsson and DiTommaso 2006). 


\section{Conclusion}

Mycorrhizal fungi play a complex role in ecosystems function, so knowledge of their distributional patterns is important especially in view of the current environmental threats to AMF diversity (Turrini and Giovannetti 2012). Our results support the hypothesis that environmental variation and host phenology can influence the AMF colonisation and spore density resulting in a shift of AMF community composition through the season. At each sampling date, there was a specific AMF community composition: some dominant AMF types were replaced by others, and some disappeared due to variation in life-history characters of the different AMF species.

Restoration of native forest may be facilitated by understanding the mycorrhizal symbioses best suited to the ecological conditions optimal for both host plant and their fungal symbionts, since different studies show contradictory results dependent on researched area. Therefore, efforts to establish the most appropriate $\mathrm{AMF}$ in culture for use in restoration programmes are needed. Optimal management of ecosystem services played by AMF could have an impact on the net gain for human society.

Acknowledgements The authors would like to thank to the Regional Directorate for Forest Resources for field sites support.

Funding information This research was financed by the Portuguese Fundação para a Ciência e a Tecnologia (PTDC/AGR-ALI/122152/ 2010, SFRH/BPD/78059/2011 and UID/GEO/04035/2019) and by the Fundo Regional para a Ciência e Tecnologia e Governo dos Açores (M3.1.7/F/010A/2009, M3.1.2/F/041/2011 and M3.1.a/F/059/2016). C. Krüger was supported by the long-term research project of the Czech Academy of Sciences RVO 67985939.

\section{Compliance with ethical standards}

Conflict of interest The authors declare that they have no conflict of interest.

Research involving human participants and/or animals This research does not contain any studies with human participants or animals.

Informed consent Informed consent is not applicable in this work.

Open Access This article is distributed under the terms of the Creative Commons Attribution 4.0 International License (http:// creativecommons.org/licenses/by/4.0/), which permits unrestricted use, distribution, and reproduction in any medium, provided you give appropriate credit to the original author(s) and the source, provide a link to the Creative Commons license, and indicate if changes were made.

\section{References}

Abdelhalim TS, Finckh MR, Babiker AG, Oehl F (2013) Species composition and diversity of arbuscular mycorrhizal fungi in White Nile state, Central Sudan. Arch Agron Soil Sci 60:377-391. https://doi. org/10.1080/03650340.2013.793453
Alguacil M d M, Díaz-Pereira E, Caravaca F et al (2009) Increased Diversity of Arbuscular Mycorrhizal Fungi in a Long-Term Field Experiment via Application of Organic Amendments to a Semiarid Degraded Soil. Appl Environ Microbiol 75:4254 4263. https://doi. org/10.1128/AEM.00316-09

Alguacil MM, Torrecillas E, García-Orenes F, Roldán A (2014) Changes in the composition and diversity of AMF communities mediated by management practices in a Mediterranean soil are related with increases in soil biological activity. Soil Biol Biochem 76:34-44. https://doi.org/10.1016/j.soilbio.2014.05.002

Alguacil MM, Torrecillas E, Lozano Z, Roldán A (2015) Arbuscular mycorrhizal fungi communities in a coral cay system (Morrocoy, Venezuela) and their relationships with environmental variables. Sci Total Environ 505:805-813. https://doi.org/10.1016/j.scitotenv. 2014.10.030

Allen SE (1989) Chemical analysis of ecological materials. Blackwell Scientific Publications, Oxford

Ávila SP, Melo C, Berning B et al (2016) Persististrombus coronatus (Mollusca: Strombidae) in the lower Pliocene of Santa Maria Island (Azores, NE Atlantic): Paleoecology, paleoclimatology and paleobiogeographic implications. Palaeogeogr Palaeoclimatol Palaeoecol 441:912-923. https://doi.org/10.1016/ j.palaeo.2015.10.043

Azevedo EB (1996) Modelação do clima insular à escala local. Modelo CIELO aplicado à ilha Terceira. Ph.D. Dissertation, University of the Azores

Azevedo EB, Santos Pereira L, Itier B (1999) Modelling the local climate in island environments: water balance applications. Agric Water Manag 40:393-403. https://doi.org/10.1016/S0378-3774(99) 00012-8

Bainard LD, Bainard JD, Hamel C, Gan Y (2014) Spatial and temporal structuring of arbuscular mycorrhizal communities is differentially influenced by abiotic factors and host crop in a semi-arid prairie agroecosystem. FEMS Microbiol Ecol 88:333-344. https://doi.org/ 10.1111/1574-6941.12300

Bati CB, Santilli E, Lombardo L (2015) Effect of arbuscular mycorrhizal fungi on growth and on micronutrient and macronutrient uptake and allocation in olive plantlets growing under high total Mn levels. Mycorrhiza 25:97-108. https://doi.org/10.1007/s00572-014-0589-0

Blaszkowski J (2012) Glomeromycota. W Szafer Institute of Botany, Polish Academy of Sciences, Krakow

Borges PAV, Aguiar C, Amaral J et al (2005) Ranking protected areas in the Azores using standardised sampling of soil epigean arthropods. Biodivers Conserv 14:2029-2060. https://doi.org/10.1007/s10531004-4283-y

Bouamri R, Dalpe Y, Serrhini MM (2014) Effect of seasonal variation on arbuscular mycorrhizal fungi associated with date palm. Emir J Food Agric:977-986. https://doi.org/10.9755/ejfa.v26i11.18985

Bouazza MK, Ighilharizn Z, de Lajudie P et al (2015) Assessing the native arbuscular mycorrhizal symbioses to rehabilitate a degraded coastal sand dune in Algeria. Int J Agric Crop Sci 8:194-202

Brigham CA, Schwartz MW (eds) (2003) Population Viability in Plants: Conservation, Management, and Modeling of Rare Plants. SpringerVerlag, Berlin Heidelberg

Chagnon P-L, Bradley RL, Maherali H, Klironomos JN (2013) A traitbased framework to understand life history of mycorrhizal fungi. Trends Plant Sci 18:484-491. https://doi.org/10.1016/j.tplants. 2013.05.001

Chaudhary VB, Lau MK, Johnson NC (2008) Macroecology of microbes-biogeography of the Glomeromycota. Mycorrhiza. Springer, In, pp 529-563

Chen K, Weixin L, Guo S et al (2012) Diversity of arbuscular mycorrhizal fungi in continuous cropping soils used for pepper production. Afr $\mathrm{J}$ Microbiol Res 6:2469-2974. https://doi.org/10.5897/AJMR11.1532

Corp IBM (2013) IBM SPSS Statistics for Windows, Version 22.0 (Version 22). IBM Corp, Armonk, NY 
Coutinho ES, Fernandes GW, Berbara RLL et al (2015) Variation of arbuscular mycorrhizal fungal communities along an altitudinal gradient in rupestrian grasslands in Brazil. Mycorrhiza 25:627-638. https://doi.org/10.1007/s00572-015-0636-5

de la Providencia IE, Nadimi M, Beaudet D et al (2013) Detection of a transient mitochondrial DNA heteroplasmy in the progeny of crossed genetically divergent isolates of arbuscular mycorrhizal fungi. New Phytol 200:211-221. https://doi.org/10.1111/nph.12372

de Novais CB, Pepe A, Siqueira JO et al (2017) Compatibility and incompatibility in hyphal anastomosis of arbuscular mycorrhizal fungi. Sci Agric 74:411-416. https://doi.org/10.1590/1678-992x2016-0243

de Oliveira JRG, de Resende GM, de Melo NF, Yano-Melo AM (2017) Symbiotic compatibility between arbuscular mycorrhizal fungi (autoctone or exotic) and three native species of the Caatinga in different phosphorus levels. Acta Sci Biol Sci 39:59-69. https:// doi.org/10.4025/actascibiolsci.v39i1.33486

Deepika S, Kothamasi D (2015) Soil moisture - a regulator of arbuscular mycorrhizal fungal community assembly and symbiotic phosphorus uptake. Mycorrhiza 25:67-75. https://doi.org/10.1007/s00572-0140596-1

Dias E (1996) Vegetação Natural dos Açores: Ecologia e Sintaxonomia das Florestas Naturais. Dissertation, University of Azores

Dumbrell AJ, Ashton PD, Aziz N et al (2011) Distinct seasonal assemblages of arbuscular mycorrhizal fungi revealed by massively parallel pyrosequencing. New Phytol 190:794-804. https://doi.org/10. $1111 /$ j.1469-8137.2010.03636.x

Egerton-Warburton LM, Johnson NC, Allen EB (2007) Mycorrhizal community dynamics following nitrogen fertilization: a cross-site test in five grasslands. Ecol Monogr 77:527-544

Elias RB, Gil A, Silva L et al (2016) Natural zonal vegetation of the Azores Islands: characterization and potential distribution. Phytocoenologia 46:107-123. https://doi.org/10.1127/phyto/2016/ 0132

Gai JP, Tian H, Yang FY et al (2012) Arbuscular mycorrhizal fungal diversity along a Tibetan elevation gradient. Pedobiologia 55:145151. https://doi.org/10.1016/j.pedobi.2011.12.004

Gilmore AE (1968) Phycomycetous mycorrhizal organisms collected by open-pot culture methods. Hilgardia 39:87-105

Gosling P, Mead A, Proctor M et al (2013) Contrasting arbuscular mycorrhizal communities colonizing different host plants show a similar response to a soil phosphorus concentration gradient. New Phytol 198:546-556. https://doi.org/10.1111/nph.12169

Graham LLB, Turjaman M, Page SE (2013) Shorea balangeran and Dyera polyphylla (syn. Dyera lowii) as tropical peat swamp forest restoration transplant species: effects of mycorrhizae and level of disturbance. Wetl Ecol Manag 21:307-321. https://doi.org/10. 1007/s11273-013-9302-x

Greipsson S, DiTommaso A (2006) Invasive non-native plants alter the occurrence of arbuscular mycorrhizal fungi and benefit from this association. Ecol Restor 24:236-241

Griffith MB, Kaufmann PR, Herlihy AT, Hill BH (2001) Analysis of Macroinvertebrate Assemblages in Relation to Environmental Gradients in Rocky Mountain Streams. Ecol Appl 11:489-505. https://doi.org/10.2307/3060904

Guo X, Gong J (2014) Differential effects of abiotic factors and host plant traits on diversity and community composition of root-colonizing arbuscular mycorrhizal fungi in a salt-stressed ecosystem. Mycorrhiza 24:79-94. https://doi.org/10.1007/s00572-013-0516-9

Hazard C, Gosling P, van der Gast CJ et al (2013) The role of local environment and geographical distance in determining community composition of arbuscular mycorrhizal fungi at the landscape scale. ISME J 7:498-508. https://doi.org/10.1038/ismej.2012.127

Hildebrandt U, Regvar M, Bothe H (2007) Arbuscular mycorrhiza and heavy metal tolerance. Phytochemistry 68:139-146. https://doi.org/ 10.1016/j.phytochem.2006.09.023
Koske RE, Walker C (1985) Species of Gigaspora (Endogonaceae) with roughened outer wall. Mycologia 77:702-720. https://doi.org/10. 1080/00275514.1985.12025159

Krüger M, Krüger C, Walker C et al (2012) Phylogenetic reference data for systematics and phylotaxonomy of arbuscular mycorrhizal fungi from phylum to species level. New Phytol 193. https://doi.org/10. $1111 / j .1469-8137.2011 .03962 . x$

Lakshmipathy R, Balakrishna AN, Bagyaraj DJ (2012) Abundance and diversity of AM fungi across a gradient of land use intensity and their seasonal variations in Niligiri Biosphere of the Western Ghats, India. J Agric Sci Technol 14:903-918

Lekberg Y, Koide RT, Rohr JR et al (2007) Role of niche restrictions and dispersal in the composition of arbuscular mycorrhizal fungal communities. J Ecol 95:95-105. https://doi.org/10.1111/j.1365-2745. 2006.01193.x

Li X, Gai J, Cai X et al (2014) Molecular diversity of arbuscular mycorrhizal fungi associated with two co-occurring perennial plant species on a Tibetan altitudinal gradient. Mycorrhiza 24:95-107. https://doi. org/10.1007/s00572-013-0518-7

Likar M, Regvar M, Mandic-Mulec I et al (2009) Diversity and seasonal variations of mycorrhiza and rhizosphere bacteria in three common plant species at the Slovenian Ljubljana Marsh. Biol Fertil Soils 45: 573-583. https://doi.org/10.1007/s00374-009-0361-3

Maherali H, Klironomos JN (2007) Influence of phylogeny on fungal community assembly and ecosystem functioning. Science 316 : 1746-1748. https://doi.org/10.1126/science.1143082

Maltz MR, Treseder KK (2015) Sources of inocula influence mycorrhizal colonization of plants in restoration projects: a meta-analysis. Restor Ecol 23:625-634. https://doi.org/10.1111/rec.12231

Martins J, Moreira O, Silva L, Moura M (2011) Vegetative propagation of the endangered Azorean tree, Picconia azorica. Arquip Life Mar Sci 28:39-46

Martins JM, Moreira OCB, Rainha NFP et al (2012) Morphophysiological dormancy and germination in seeds of the Azorean tree Picconia azorica. Seed Sci Technol 40:163-176. https://doi.org/10.15258/sst.2012.40.2.02

Martins JM, Moreira OCB, Sardos J et al (2013) Population genetics and conservation of the Azorean tree Picconia azorica. Biochem Syst Ecol 49:135-143. https://doi.org/10.1016/j.bse.2013.03.027

McGonigle TP, Miller MH, Evans DG et al (1990) A new method which gives an objective measure of colonization of roots by vesiculararbuscular mycorrhizal fungi. New Phytol 115:495-501. https://doi. org/10.1111/j.1469-8137.1990.tb00476.x

Melo CD, Walker C, Rodríguez-Echeverría S et al (2014) Species composition of arbuscular mycorrhizal fungi differ in semi-natural and intensively managed pastures in an isolated oceanic island (Terceira, Azores). Symbiosis 64:73-85. https://doi.org/10.1007/ s13199-014-0303-1

Melo CD, Luna S, Krüger C et al (2017) Arbuscular mycorrhizal fungal community composition associated with Juniperus brevifolia in native Azorean forest. Acta Oecologica 79:48-61. https://doi.org/10. 1016/j.actao.2016.12.006

Melo CD, Luna S, Krüger C et al (2018) Communities of arbuscular mycorrhizal fungi under Picconia azorica in native forests of Azores. Symbiosis 74:43-54. https://doi.org/10.1007/s13199017-0487-2

Minitab I (2000) Minitab: release 13 for Windows. Minitab Inc., State College, PA

Miranda JCCD, Harris PJ (1994) Effects of soil phosphorus on spore germination and hyphal growth of arbuscular mycorrhizal fungi. New Phytol 128:103-108. https://doi.org/10.1111/j.1469-8137. 1994.tb03992.x

Moora M, Davison J, Öpik M et al (2014) Anthropogenic land use shapes the composition and phylogenetic structure of soil arbuscular mycorrhizal fungal communities. FEMS Microbiol Ecol 90:609-621. https://doi.org/10.1111/1574-6941.12420 
Olsen SR, Sommers LE (1982) Phosphorus. In: Page AL, Miller RH, Keeney DR (eds) Methods of soil analysis. American Soc. Of Agronomy, Madison, Wisconsin, USA, pp 403-427

Öpik M, Moora M, Liira J, Zobel M (2006) Composition of rootcolonizing arbuscular mycorrhizal fungal communities in different ecosystems around the globe: Arbuscular mycorrhizal fungal communities around the globe. J Ecol 94:778-790. https://oi.org/10. 1111/j.1365-2745.2006.01136.x

Pande M, Tarafdar JC (2004) Arbuscular mycorrhizal fungal diversity in neem-based agroforestry systems in Rajasthan. Appl Soil Ecol 26: 233-241. https://doi.org/10.1016/j.apsoil.2003.12.009

Panwar V, Meghvansi MK, Siddiqui S (2011) Short-term temporal variation in sporulation dynamics of arbuscular mycorrhizal (AM) fungi and physico-chemical edaphic properties of wheat rhizosphere. Saudi J Biol Sci 18:247-254. https://doi.org/10.1016/j. sjbs.2010.12.012

Porras-Soriano A, Soriano-Martín ML, Porras-Piedra A, Azcón R (2009) Arbuscular mycorrhizal fungi increased growth, nutrient uptake and tolerance to salinity in olive trees under nursery conditions. J Plant Physiol 166:1350-1359. https://doi.org/10.1016/j. jplph.2009.02.010

Powell JR, Parrent JL, Hart MM et al (2009) Phylogenetic trait conservatism and the evolution of functional trade-offs in arbuscular mycorrhizal fungi. Proc R Soc B Biol Sci 276:4237-4245. https://doi. org/10.1098/rspb.2009.1015

Pozo MJ, Azcón-Aguilar C (2007) Unraveling mycorrhiza-induced resistance. Curr Opin Plant Biol 10:393-398. https://doi.org/10.1016/j. pbi.2007.05.004

Redecker D, Schüßler A, Stockinger H et al (2013) An evidence-based consensus for the classification of arbuscular mycorrhizal fungi (Glomeromycota). Mycorrhiza 23:515-531. https://doi.org/10. 1007/s00572-013-0486-y

Rillig MC, Mummey DL (2006) Mycorrhizas and soil structure. New Phytol 171:41-53. https://doi.org/10.1111/j.1469-8137.2006. 01750.x

Schalamuk S, Cabello M (2010) Arbuscular mycorrhizal fungal propagules from tillage and no-tillage systems: possible effects on Glomeromycota diversity. Mycologia 102:261-268. https://doi. org/10.3852/08-118

Schüßler A, Walker C (2019) Archaeospora ecuadoriana sp. nov. from a mountainous biodiversity hotspot area in Ecuador, and transfer of Palaeospora spainiae to Archaeospora, as A. spainiae comb. nov. Mycorrhiza. https://doi.org/10.1007/s00572-019-00913-2

Schüßler A, Schwarzott D, Walker C (2001) A new fungal phylum, the Glomeromycota: phylogeny and evolution. Mycol Res 105:14131421

Schüßler A, Krüger M, Walker C (2011) Revealing Natural Relationships among Arbuscular Mycorrhizal Fungi: Culture Line BEG47 Represents Diversispora epigaea, Not Glomus versiforme. PLoS One 6(e23333):1-12. https://doi.org/10.1371/journal.pone.0023333

Seaby RMH, Henderson PA, Prendergast JR (2004) Community Analysis Package. Version 4.01. Pisces Conservation Ltd., http:// www.pisces-conservation.com

Senés-Guerrero C, Schüßler A (2016) A conserved arbuscular mycorrhizal fungal core-species community colonizes potato roots in the Andes. Fungal Divers 77:317-333. https://doi.org/10.1007/ s13225-015-0328-7

Silva L, Martins M, Maciel G, Moura M (2009) Flora Vascular dos Açores - Prioridades em Conservação. Azorean Vascular Flora Priorities in Conservation. Bilingue. Associação Ecológica Amigos dos Açores \& CCPA, Ponta Delgada

Silva L, Moura M, Schaefer H et al (2010) A List of the Terrestrial and Marine Biota from the Azores. In: Costa A, Cunha R et al (eds) Borges PAV. Princípia, Cascais, pp 117-163
Soka G, Ritchie M (2014) Arbuscular mycorrhizal symbiosis and ecosystem processes: Prospects for future research in tropical soils. Open J Ecol 4:11-22. https://doi.org/10.4236/oje.2014.41002

Sun X, Su Y, Zhang Y et al (2013) Diversity of arbuscular mycorrhizal fungal spore communities and its relations to plants under increased temperature and precipitation in a natural grassland. Chin Sci Bull 58:4109-4119. https://doi.org/10.1007/s11434-013-5961-5

ter Braak CJF, Smilauer P (2002) CANOCO reference manual and CanoDraw for Windows user's guide: software for canonical ordination, version 4.5. Microcomputer Power, Ithaca, NewYork

ter Braak CJF, Wiertz J (1994) On the Statistical Analysis of Vegetation Change: A Wetland Affected by Water Extraction and Soil Acidification. J Veg Sci 5:361-372. https://doi.org/10.2307/ 3235860

Torrecillas E, Torres P, Alguacil MM et al (2013) Influence of Habitat and Climate Variables on Arbuscular Mycorrhizal Fungus Community Distribution, as Revealed by a Case Study of Facultative Plant Epiphytism under Semiarid Conditions. Appl Environ Microbiol 79:7203-7209. https://doi.org/10.1128/AEM.02466-13

Trabelsi D, Mhamdi R (2013) Microbial Inoculants and Their Impact on Soil Microbial Communities: A Review. BioMed Res Int 2013:111. https://doi.org/10.1155/2013/863240

Triantis KA, Borges PAV, Ladle RJ et al (2010) Extinction debt on oceanic islands. Ecography 33:285-294. https://doi.org/10.1111/j. 1600-0587.2010.06203.x

Turrini A, Giovannetti M (2012) Arbuscular mycorrhizal fungi in national parks, nature reserves and protected areas worldwide: a strategic perspective for their in situ conservation. Mycorrhiza 22:81-97. https://doi.org/10.1007/s00572-011-0419-6

Turrini A, Sbrana C, Strani P et al (2010) Arbuscular mycorrhizal fungi of a Mediterranean island (Pianosa), within a UNESCO Biosphere Reserve. Biol Fertil Soils 46:511-520. https://doi.org/10.1007/ s00374-010-0446-Z

van der Gast CJ, Gosling P, Tiwari B, Bending GD (2011) Spatial scaling of arbuscular mycorrhizal fungal diversity is affected by farming practice. Environ Microbiol 13:241-249. https://doi.org/10.1111/j. 1462-2920.2010.02326.x

Van der Heijden MGA, Scheublin TR (2007) Functional traits in mycorrhizal ecology: their use for predicting the impact of arbuscular mycorrhizal fungal communities on plant growth and ecosystem functioning. New Phytol 174:244-250. https://doi.org/10.1111/j. 1469-8137.2007.02041.x

Varela-Cervero S, López-García Á, Barea JM, Azcón-Aguilar C (2016) Differences in the composition of arbuscular mycorrhizal fungal communities promoted by different propagule forms from a Mediterranean shrubland. Mycorrhiza 26:489-496. https://doi.org/ 10.1007/s00572-016-0687-2

Velázquez MS, Cabello MN, Barrera M (2013) Composition and structure of arbuscular-mycorrhizal communities in El Palmar National Park, Argentina. Mycologia 105:509-520. https://doi.org/10. 3852/11-353

Velázquez MS, Stürmer SL, Bruzone C et al (2016) Occurrence of arbuscular mycorrhizal fungi in high altitude sites of the Patagonian Altoandina region in Nahuel Huapi National Park (Argentina). Acta Bot Bras 30:521-531. https://doi.org/10.1590/ 0102-33062016abb0223

Velázquez MS, Fabisik JC, Abarca CL et al (2018) Colonization dynamics of arbuscular mycorrhizal fungi (AMF) in Ilex paraguariensis crops: Seasonality and influence of management practices. J King Saud Univ - Sci. https://doi.org/10.1016/j.jksus.2018.03.017

Walker C (1992) Systematics and taxonomy of the arbuscular endomycorrhizal fungi (Glomales)- a possible way forward. Agronomie 12:887-897

Walker C, Diederichs C (1989) Scutellospora scutata sp. nov., A newly described endomycorrhizal fungus from Brazil. Mycotaxon 35: $357-361$ 
Walker C, Trappe JM (1981) Acaulospora spinosa sp. nov. with a key to the species of Acaulospora. Mycotaxon 12:515-521

Walker C, Vestberg M (1998) Synonymy amongst the arbuscular mycorrhizal fungi: Glomus claroideum, G. maculosum, G. multisubstenum and G. fistulosum. Ann Bot 82:601-624. https:// doi.org/10.1006/anbo.1998.0714

Walker C, Reed L, Sanders FE (1984) Acaulospora nicolsonii, a new endogonaceous species from Great Britain. Trans Br Mycol Soc 83:360-364

Walker C, Pfeiffer CM, Bloss HE (1986) Acaulospora delicata sp. Nov. An endomycorrhizal fungus from Arizona. Mycotaxon 25:621-628

Wang M-Y, Ren-Xue X, Qiang-Sheng W et al (2007) Influence of arbuscular mycorrhizal fungi on microbes and enzymes of soils from different cultivated densities of red clover. Ann Microbiol 57:1-7. https://doi.org/10.1007/BF03175042

Wang C, Gu Z, Cui H et al (2015) Differences in Arbuscular Mycorrhizal Fungal Community Composition in Soils of Three Land Use Types in Subtropical Hilly Area of Southern China. PloS One 10: e0130983
Willis A, Rodrigues BF, Harris PJC (2013) The Ecology of Arbuscular Mycorrhizal Fungi. Crit Rev Plant Sci 32:1-20. https://doi.org/10. 1080/07352689.2012.683375

Zhang J, Wang F, Che R et al (2016) Precipitation shapes communities of arbuscular mycorrhizal fungi in Tibetan alpine steppe. Sci Rep 6: 23488. https://doi.org/10.1038/srep23488

Zhang H, Wei S, Hu W et al (2017) Arbuscular Mycorrhizal Fungus Rhizophagus irregularis Increased Potassium Content and Expression of Genes Encoding Potassium Channels in Lycium barbarum. Front Plant Sci 8:440. https://doi.org/10.3389/fpls. 2017.00440

Zhao H, Li X, Zhang Z et al (2017) Species diversity and drivers of arbuscular mycorrhizal fungal communities in a semi-arid mountain in China. PeerJ 5. https://doi.org/10.7717/peerj.4155

Publisher's note Springer Nature remains neutral with regard to jurisdictional claims in published maps and institutional affiliations. 